ALEA, Lat. Am. J. Probab. Math. Stat. 14, 631-656 (2017)

DOI: 10.30757/ALEA.v14-31

\title{
Weingarten calculus via orthogonality relations: new applications
}

\author{
Benoît Collins and Sho Matsumoto \\ Department of Mathematics, Kyoto University \\ Kitashirakawa Oiwake-cho, Sakyo-ku, Kyoto 606-8502, Japan. \\ E-mail address: collins@math.kyoto-u.ac.jp \\ Graduate School of Science and Engineering, Kagoshima University \\ 1-21-35, Korimoto, Kagoshima 890-0065, Japan. \\ E-mail address: shom@sci.kagoshima-u.ac.jp
}

\begin{abstract}
Weingarten calculus is a completely general and explicit method to compute the moments of the Haar measure on compact subgroups of matrix algebras. Particular cases of this calculus were initiated by theoretical physicists - including Weingarten, after whom this calculus was coined by the first author, after investigating it systematically. Substantial progress was achieved subsequently by the second author and coworkers, based on representation theoretic and combinatorial techniques. All formulas of 'Weingarten calculus' are in the spirit of Weingarten's seminal paper (Weingarten, 1978). However, modern proofs are very different from Weingarten's initial ideas. In this paper, we revisit Weingarten's initial proof and we illustrate its power by uncovering two new important applications: (i) a uniform bound on the Weingarten function, that subsumes existing uniform bounds, and is optimal up to a polynomial factor, and (ii) an extension of Weingarten calculus to symmetric spaces and conceptual proofs of identities established by the second author.
\end{abstract}

\section{Introduction}

Let $G$ be a compact subgroup of $M_{d}(\mathbb{C}), \mu$ its probability Haar measure, and $u_{i j}: G \rightarrow \mathbb{C}$ the $i j$-coordinate function. A Weingarten type formula is a formula that computes $\int_{G} u_{i_{1} j_{1}} \ldots d \mu$. It is in general given as a sum over conditions on the indices $i, j$ 's, of functions called Weingarten functions. For example, in the case of unitary groups, the conditions are labeled by permutations, and in the orthogonal group, they are given by pair partitions. We refer to sections 2.1 and 4.1 for details.

Received by the editors January 19th, 2017; accepted February 17th, 2017.

2010 Mathematics Subject Classification. 60B20, 15B52, 05 A05.

Key words and phrases. Random matrix, Weingarten calculus, Haar measure, compact Lie group, circular orthogonal ensemble, compact symmetric space.

Research supported by JSPS KAKENHI Grant Numbers JP26800048, JP25800062. 
Weingarten's initial motivation (Weingarten, 1978) was to consider a sequence of subgroups $G_{d}$ of $M_{d}(\mathbb{C})$, typically the unitary or orthogonal groups, and rather than describing precisely the integration formula, he was interested in the large $d$ asymptotics of integrals.

His basic observation was that the Weingarten functions satisfies a family of linear equations, and under an appropriate rescaling of the Weingarten functions by polynomials in $d$, they were satisfying a system that was upper triangular in the large $d$ limit, and therefore, invertible for $d$ large enough. This approach was very slick, but one drawback was that it did not give precise information on the size of $d$ for which there was a unique solution. Until very recently, this idea has been dropped and replaced by equations arising from representation theory Collins (2003); Collins and Śniady (2006); Matsumoto and Novak (2013) and others. Another drawback is that the concrete methods to solve this linear system were not developed. The purpose of this paper is to revisit Weingarten's original approach, and address the aforementioned drawbacks.

Specifically, the first author, together with Brannan, in Brannan and Collins (2016), realized recently that Weingarten's original approach was unavoidable when dealing with the asymptotics of Weingarten functions in the case of compact quantum groups, and could be improved into a very powerful and conceptual tool in this context.

In this paper, we revisit this tool in the context of the classical group. The formulas that one obtains are closely related to results obtained in Matsumoto and Novak (2013), but they are more elementary and more general, in the sense that no knowledge on Jucys-Murphy elements is needed, and that the technique can be adapted to more general cases.

Weingarten calculus has proven very useful in many situations, including free probability, random matrix theory, quantum information theory, representation theory, matrix integrals, and others (we refer to most of the recent items of the bibliography for applications). One of the strength of this calculus is the very interesting properties of the Weingarten function in the large $d$ limit. For example, in the unitary case,

$$
\mathrm{Wg}(\sigma, d)=d^{-k-|\sigma|} \operatorname{Moeb}(\sigma)
$$

We refer for section 3 for notation.

In some cases, it is desirable to obtain uniform estimates on Wg. Such bounds have been obtained by the first author and other coauthors in Collins et al. (2013, Theorem 4.1), and also by Montanaro (2013, Lemma 16).

In a related domain, in random matrix theory, moment methods are a widely used and powerful tool. On the one hand, the proofs of asymptotic freeness of unitarily invariant random matrix under the weakest possible assumptions are obtained with Weingarten techniques (Collins, 2003). On the other hand, uniform moment estimates of a power or a random matrix degree depending on the dimension, and high enough (typically much higher than the logarithm of the dimension) give norm convergence estimates. We refer to Soshnikov (1999) for one of the first seminal applications of this method to random matrix theory.

Putting these two observations together, it is very natural to try to achieve a fine and uniform convergence of the behaviour of the Weingarten function as the dimension goes to infinity, and the size of the permutation group too. This problem of finding a uniform estimate has also applications in more unexpected fields, such 
as Quantum Information Theory, cf e.g. Montanaro (2013); Collins et al. (2013) for weaker uniform bounds with specific applications. See also Berkolaiko and Kuipers (2013).

In this respect, our main result is a uniform bound within a polynomial factor, which is obtained in Theorems 3.2, 4.10, and 4.11 and which we record in the theorem below just in the case of the unitary group - the other cases covered in this paper showcase results of similar flavour (albeit with different proofs)

Theorem 1.1. For any $\sigma \in \mathcal{S}_{k}$ and $d>\sqrt{6} k^{7 / 4}$,

$$
\frac{1}{1-\frac{k-1}{d^{2}}} \leq \frac{d^{k+|\sigma|} \mathrm{Wg}^{\mathrm{U}}(\sigma, d)}{\operatorname{Moeb}(\sigma)} \leq \frac{1}{1-\frac{6 k^{7 / 2}}{d^{2}}}
$$

In addition, the l.h.s inequality is valid for any $d \geq k$.

Let us note that this revisited approach to Weingarten calculus is related to, and implies results of Matsumoto and Novak (2013) in the unitary case and from Zinn-Justin (2010); Matsumoto (2011) in the orthogonal case.

Finally, Weingarten calculus extends beyond groups, to the context of symmetric spaces Cartan (1927). Although push forward allow in principle to compute any Haar measure on a symmetric space Collins and Stolz (2008), the second author observed some phenomena intrinsic to some classes Matsumoto (2012, 2013). These phenomena were obtained by computation without conceptual explanation. It turns out that in some cases, Weingarten's original approach supplies this conceptual explanation. This is the content of theorems 5.4 and 5.7.

This paper is organized as follows: After this introduction, section 2 revisits and conceptualizes Weingarten's original integration technique in the unitary context. Section 3 uses section 2 to provide the best uniform bounds known so far. Section 4 handles sections 2 and 3 in the context of orthogonal and symplectic cases. Section 5 develops the Weingarten calculus on symmetric spaces.

\section{Unitary groups}

2.1. Weingarten calculus. Throughout this section, we suppose $d, k$ are positive integers with $d \geq k$. For each permutation $\sigma \in \mathcal{S}_{k}$, the unitary Weingarten function $\mathrm{Wg}^{\mathrm{U}}(\sigma, d)$ is, by definition,

$$
\mathrm{Wg}^{\mathrm{U}}(\sigma, d)=\int_{\mathrm{U}(d)} u_{11} u_{22} \cdots u_{k k} \overline{u_{\sigma(1) 1} u_{\sigma(2) 2} \cdots u_{\sigma(k) k}} d \mu,
$$

where $d \mu=d \mu^{\mathrm{U}(d)}$ denotes the normalized Haar measure on $\mathrm{U}(d)$. It is easy to see that the function $\sigma \mapsto \mathrm{Wg}^{\mathrm{U}}(\sigma, d)$ is conjugacy-invariant, i.e.,

$$
\mathrm{Wg}^{\mathrm{U}}\left(\tau^{-1} \sigma \tau, d\right)=\mathrm{Wg}^{\mathrm{U}}(\sigma, d) \quad \text { for any } \sigma, \tau \in \mathcal{S}_{k} \text {. }
$$

The Weingarten calculus for $\mathrm{U}(d)$ is described as follows.

Lemma 2.1 (Collins, 2003). For four sequences

$$
\mathbf{i}=\left(i_{1}, \ldots, i_{k}\right), \quad \mathbf{i}^{\prime}=\left(i_{1}^{\prime}, \ldots, i_{k}^{\prime}\right), \quad \mathbf{j}=\left(j_{1}, \ldots, j_{k}\right), \quad \mathbf{j}^{\prime}=\left(j_{1}^{\prime}, \ldots, j_{k}^{\prime}\right)
$$


of positive integers in $\{1,2, \ldots, d\}$, we have

$$
\begin{aligned}
& \int_{\mathrm{U}(d)} u_{i_{1}, j_{1}} u_{i_{2}, j_{2}} \cdots u_{i_{k}, j_{k}} \overline{u_{i_{1}^{\prime}, j_{1}^{\prime}} u_{i_{2}^{\prime}, j_{2}^{\prime}} \cdots u_{i_{k}^{\prime}, j_{k}^{\prime}}} d \mu \\
= & \sum_{\sigma \in \mathcal{S}_{k}} \sum_{\tau \in \mathcal{S}_{k}} \delta_{\sigma}\left(\mathbf{i}, \mathbf{i}^{\prime}\right) \delta_{\tau}\left(\mathbf{j}, \mathbf{j}^{\prime}\right) \mathrm{Wg}^{\mathrm{U}}\left(\sigma \tau^{-1}, d\right) .
\end{aligned}
$$

Here $\delta_{\sigma}\left(\mathbf{i}, \mathbf{i}^{\prime}\right)$ is given by

$$
\delta_{\sigma}\left(\mathbf{i}, \mathbf{i}^{\prime}\right)= \begin{cases}1 & \text { if } i_{\sigma(r)}=i_{r}^{\prime} \text { for all } r \\ 0 & \text { otherwise }\end{cases}
$$

2.2. Orthogonality relations. We give orthogonality relations for Weingarten functions $\mathrm{Wg}^{\mathrm{U}}(\cdot, d)$, which comes from the orthogonal (or unitary) property of the random matrix $U$ itself. This is first found in Samuel (1980). See also Goulden et al. (2013) and its references.

Proposition 2.2. For any $\sigma \in \mathcal{S}_{k}$, we have

$$
d \mathrm{Wg}^{\mathrm{U}}(\sigma, d)=-\sum_{i=1}^{k-1} \mathrm{Wg}^{\mathrm{U}}((i, k) \sigma, d)+\delta_{\sigma(k)=k} \mathrm{Wg}^{\mathrm{U}}\left(\sigma^{\downarrow}, d\right) .
$$

Here $\sigma^{\downarrow} \in \mathcal{S}_{k-1}$ is the restriction of $\sigma$ to the permutation on the set $\{1,2, \ldots, k-1\}$ (if $\sigma(k)=k$ ) and $(i, k)$ is the transposition between $i$ and $k$. Moreover, $\delta_{\sigma(k)=k}$ equals to 1 if $\sigma$ fixes $k$, and to 0 otherwise.

Proof: Consider the sum of integrals

$$
\sum_{i=1}^{d} \int_{\mathrm{U}(d)} u_{11} \cdots u_{k-1, k-1} u_{k, i} \overline{u_{\sigma(1) 1} \cdots u_{\sigma(k-1), k-1} u_{\sigma(k), i}} d \mu .
$$

Since a matrix $U=\left(u_{i j}\right)$ is unitary, we have $\sum_{i=1}^{d} u_{k, i} \overline{u_{\sigma(k), i}}=\delta_{\sigma(k)=k}$ and therefore it equals

$$
\begin{aligned}
& \delta_{\sigma(k)=k} \int_{\mathrm{U}(d)} u_{11} \cdots u_{k-1, k-1} \overline{u_{\sigma(1) 1} \cdots u_{\sigma(k-1), k-1}} d \mu \\
= & \delta_{\sigma(k)=k} \operatorname{Wg}^{\mathrm{U}}\left(\sigma^{\downarrow}, d\right) .
\end{aligned}
$$

On the other hand, using Lemma 2.1 we have

$$
\begin{aligned}
& \int_{\mathrm{U}(d)} u_{11} \cdots u_{k-1, k-1} u_{k, i} \overline{u_{\sigma(1) 1} \cdots u_{\sigma(k-1), k-1} u_{\sigma(k), i}} d \mu \\
= & \begin{cases}\operatorname{Wg}^{\mathrm{U}}(\sigma, d) & \text { if } i \geq k, \\
\operatorname{Wg}^{\mathrm{U}}(\sigma, d)+\mathrm{Wg}^{\mathrm{U}}((i, k) \sigma, d) & \text { if } i<k .\end{cases}
\end{aligned}
$$

In fact, in the notation of Lemma 2.1 , the delta symbol $\delta_{\tau}\left(\mathbf{j}, \mathbf{j}^{\prime}\right)$ with $\mathbf{j}=\mathbf{j}^{\prime}=$ $(1, \ldots, k-1, i)$ survives only if $\tau$ is the identity permutation or the transposition $(i, k)$ with $i<k$. Summing up them over $i$, we obtain

$$
(2.3)=d \mathrm{Wg}^{\mathrm{U}}(\sigma, d)+\sum_{i=1}^{k-1} \mathrm{Wg}^{\mathrm{U}}((i, k) \sigma, d)
$$

Combining this with (2.4), we obtain the proposition. 
Example 2.3. We use the one-row notation $[\sigma(1), \sigma(2), \ldots, \sigma(k)]$ for $\sigma \in \mathcal{S}_{k}$. The relation (2.2) with $k=1$ and $\sigma=[1] \in \mathcal{S}_{1}$ gives the relation $d \mathrm{Wg}^{\mathrm{U}}([1], d)=$ $\mathrm{Wg}^{\mathrm{U}}(\emptyset, d)=1$, and hence $\mathrm{Wg}^{\mathrm{U}}([1], d)=\frac{1}{d}$. Furthermore, for $k=2$ we find

$$
\begin{aligned}
& d \mathrm{Wg}^{\mathrm{U}}([1,2], d)=-\mathrm{Wg}^{\mathrm{U}}([2,1], d)+\mathrm{Wg}^{\mathrm{U}}([1], d), \\
& d \mathrm{Wg}^{\mathrm{U}}([2,1], d)=-\mathrm{Wg}^{\mathrm{U}}([1,2], d) .
\end{aligned}
$$

Solving this linear system of equations, we obtain

$$
\mathrm{Wg}^{\mathrm{U}}([1,2], d)=\frac{1}{d^{2}-1}, \quad \mathrm{Wg}^{\mathrm{U}}([2,1], d)=\frac{-1}{d\left(d^{2}-1\right)} .
$$

\subsection{Weingarten graphs.}

Definition 2.4. We define an infinite directed graph $\mathcal{G}^{\mathrm{U}}=(V, E)$ as follows.

- The vertex set $V$ is $\bigsqcup_{k=0}^{\infty} \mathcal{S}_{k}$. Each vertex $v$ in $\mathcal{S}_{k}$ is said to be of level $k$. The vertex $\emptyset \in \mathcal{S}_{0}$ is the unique element of level 0 .

- For each $k \geq 2$, two vertices $\sigma, \tau$ of level $k$ (i.e. in $\mathcal{S}_{k}$ ) are connected by a solid arrow as $\sigma \rightarrow \tau$ if and only if

$$
\tau=(i, k) \sigma \quad \text { with some } i \text { smaller than } k \text {. }
$$

- For each $k \geq 1$, a vertex $\sigma$ of level $k$ and a vertex $\sigma^{\prime}$ of level $k-1$ are connected by a dashed arrow as $\sigma \rightarrow \sigma^{\prime}$ if and only if $\sigma(k)=k$ and $\sigma^{\prime}=\sigma^{\downarrow}$.

We call $\mathcal{G}^{\mathrm{U}}$ the Weingarten graph for the unitary group.

Each vertex $\sigma$ of level $k$ is connected by exactly $k-1$ solid arrows and radiates at most 1 dashed arrow to $\sigma^{\downarrow}$ if it exists.

Let $\sigma$ be a vertex of level $k$. A sequence $p=\left(\sigma_{0}, \sigma_{1}, \ldots, \sigma_{l+k}\right)$ of vertices is called a path from $\sigma$ to $\emptyset$ of length $l+k$ if $\sigma_{0}=\sigma, \sigma_{l+k}=\emptyset$, and, for each $i=1,2, \ldots, l+k$, vertices $\sigma_{i-1}$ and $\sigma_{i}$ are connected by an edge. Since only dashed arrows lower levels of vertices, a path $p$ always goes through exactly $k$ dashed arrows, and hence the length of $p$ is at least $k$. Denote by $P(\sigma, l)$ the collection of such paths. Especially, every path $p \in P(\sigma, l)$ goes through exactly $l$ solid edges.

Lemma 2.5. For $\sigma \in \mathcal{S}_{k}$, we have the expansion

$$
\mathrm{Wg}^{\mathrm{U}}(\sigma, d)=d^{-k} \sum_{l \geq 0} \# P(\sigma, l)\left(-d^{-1}\right)^{l} .
$$

Proof: The relation (2.2) is expressed as

$$
\mathrm{Wg}^{\mathrm{U}}(\sigma, d)=\sum_{\tau: \sigma \rightarrow \tau} \mathrm{Wg}^{\mathrm{U}}(\tau, d)\left(-d^{-1}\right)+\delta_{\sigma(k)=k} \mathrm{Wg}^{\mathrm{U}}\left(\sigma^{\downarrow}, d\right) d^{-1},
$$

where the sum of the right hand side runs over $\tau \in \mathcal{S}_{k}$ connected with $\sigma$ by a solid arrow.

We consider the infinite dimensional vector space spanned by the basis $V$ and we denote it $\mathbb{C}^{V}$. We call $\delta_{\sigma}, \sigma \in \mathcal{S}_{k}$. On $\mathbb{C}^{V}$ we introduce the linear map that is the linear extension of

$$
Q\left(\delta_{\sigma}\right)= \begin{cases}\sum_{\tau: \sigma \rightarrow \tau}\left(-d^{-1}\right) \delta_{\tau}+\delta_{\sigma(k)=k} d^{-1} \delta_{\sigma \downarrow} & \text { if } \sigma \neq \emptyset \\ \delta_{\emptyset} & \text { if } \sigma=\emptyset .\end{cases}
$$


In addition, let us introduce the linear form $\mathrm{Wg}: \mathbb{C}^{V} \rightarrow \mathbb{C}$ given by $\mathrm{Wg}\left(\delta_{\sigma}\right)=$ $\mathrm{Wg}^{\mathrm{U}}(\sigma, d)$. It follows directly from equation (2.2) that

$$
\mathrm{Wg} \circ Q=\mathrm{Wg} \text {. }
$$

Note that $Q\left(\delta_{\emptyset}\right)=\delta_{\emptyset}$ but for any other basis element, $Q$ has the effect of multiplying by $d^{-1}$ times a vector whose coordinates do not depend on $d$. More precisely, if we view $Q$ formally as an endomorphism of $\mathbb{C}\left[\left[d^{-1}\right]\right]^{V}$, we can write it as

$$
Q=P+d^{-1} T
$$

where $P, T$ are endomorphisms of $\mathbb{C}^{V}$ (that act naturally on $\mathbb{C}\left[\left[d^{-1}\right]\right]^{V}$ ), $P$ is the rank one projection whose range is $\mathbb{C} \delta_{\emptyset}$ and whose kernel is the span of all remaining canonical basis elements.

Therefore, it follows directly that $Q^{\circ l}\left(\delta_{\sigma}\right)$ converges formally as $l \rightarrow \infty$ (in the sense that each coefficient $Q^{\circ l}\left(\delta_{\sigma}\right)_{\tau}$ (of $Q^{\circ l}\left(\delta_{\sigma}\right)$ ) viewed as a rational fraction in $d^{-1}$ converges pointwise - specifically, the term of degree $p$ becomes steady as soon as $l>p$ because of the structure of $Q$. In this sense, we can define the limit of $Q^{\circ l}$ as $l \rightarrow \infty$ as an element of $\operatorname{End}\left(\mathbb{C}\left[\left[d^{-1}\right]\right]^{V}\right)$, that we will call $Q^{\circ \infty}$.

If one knows beforehand that $\mathrm{Wg}^{\mathrm{U}}(\sigma, d)$ can be seen as a power series in $d^{-1}$ (this is the case because it is rational fraction) then the proof is complete by considering the limit $Q^{\circ \infty}$ of $Q^{\circ l}$ as $l \rightarrow \infty$, and the equation $\mathrm{Wg} \circ Q^{\circ \infty}=\mathrm{Wg}$ at $\delta_{\sigma}$.

However, for the sake of completeness and of obtaining more information on $\mathrm{Wg}$, we add one analytic proof that does not require the knowledge that $\mathrm{Wg}^{\mathrm{U}}(\sigma, d)$ is a power series in $d^{-1}$.

For this, we introduce the subset $V_{n}$ of $V$ as the finite disjoint union of the $n+1$ first symmetric groups $V_{n}=\bigsqcup_{k=0}^{n} \mathcal{S}_{k}$. It is clear that $\mathrm{Wg}$ can be defined on $\mathbb{C}^{V_{n}} \subset \mathbb{C}^{V}$ and that $Q$ leaves $\mathbb{C}^{V_{n}}$ invariant, and that on $\mathbb{C}^{V_{n}}, \mathrm{Wg} \circ Q^{\circ \infty}=\mathrm{Wg}$ remains true on $\mathbb{C}^{V_{n}}$, we introduce the $l_{1}$-type norm $\left\|\sum \alpha_{\tau} \delta_{\tau}\right\|=\sum\left|\alpha_{\tau}\right|$.

We use the notation $P$ for the projection introduced earlier in the first part of this proof, and we note that $Q \circ P=P$. Next, we introduce the notation $R=Q \circ(1-P)$. Note that $Q=P+R$. By inspecting equation (2.2) one sees that

$$
\|R(x)\| \leq n d^{-1}\|x\| .
$$

Iterating, for any integer $l \geq 1,\left\|R^{\circ l}(x)\right\| \leq n^{l} d^{-l}\|x\|$. One checks by induction that for any integer $l>1$,

$$
Q^{\circ l} \circ(1-P)=P \circ\left(R+R^{\circ 2}+\ldots+R^{\circ l-1}\right)+R^{\circ k} .
$$

Therefore,

$$
Q^{\circ l}=P \circ\left(1+R+R^{\circ 2}+\ldots+R^{\circ l-1}\right)+R^{\circ k} .
$$

The inequality $\left\|R^{\circ l}(x)\right\| \leq n^{l} d^{-l}\|x\|$ implies $\left\|P \circ R^{\circ l}(x)\right\| \leq n^{l} d^{-l}\|x\|$, therefore $Q^{\circ l}$ converges with respect to any norm topology (as they are all equivalent in finite dimension).

Calling again its limit $Q^{\circ \infty}$, we conclude that $\mathrm{Wg} \circ Q^{\circ \infty}=\mathrm{Wg}$, apply this equality at $\delta_{\sigma}$, and conclude as in the formal case.

Remark 2.6. As a byproduct of the analytic proof presented above, we obtain a bound on the Weingarten function for any $d>n$. This bound is refined and uniformized subsequently in this paper. 
For each permutation $\sigma \in \mathcal{S}_{k}$, we associate with the cycle-type $\mu$, which is an integer partition of $k$. Put $|\sigma|=k-\ell(\mu) \in\{0,1, \ldots, k-1\}$, where $\ell(\mu)$ is the length of $\mu$. For example,

$$
|\sigma|= \begin{cases}0 & \text { if } \sigma \text { is the identity permutation, } \\ 1 & \text { if } \sigma \text { is a transposition, }\end{cases}
$$

and $|\sigma| \geq 2$ otherwise.

For any transposition $(i, j)$, we find that $|(i, j) \sigma|$ is equal to $|\sigma| \pm 1$. Moreover, if $\sigma(k)=k$, then $\left|\sigma^{\downarrow}\right|=|\sigma|$. In other words, for a path $p=\left(\sigma_{0}, \sigma_{1}, \ldots, \sigma_{l+k}\right)$ in $P(\sigma, l)$, we see that

$$
\left|\sigma_{i}\right|-\left|\sigma_{i-1}\right|= \begin{cases}+1 \text { or }-1 & \text { if } \sigma_{i-1} \longrightarrow \sigma_{i}, \\ 0 & \text { if } \sigma_{i-1}-\rightarrow \sigma_{i} .\end{cases}
$$

Since $\left|\sigma_{l+k}\right|=|\emptyset|=0$, we find \#P( $\left.\sigma, l\right)=0$ unless $l=|\sigma|+2 g$ with some integer $g \geq 0$. We call this property a parity condition for path $p$ (or for $\sigma$ ). The expansion (2.5) can be now reformulated as follows.

Theorem 2.7. For each $\sigma \in \mathcal{S}_{k}$, we have the formal expansion

$$
(-1)^{|\sigma|} d^{|\sigma|+k} \mathrm{Wg}^{\mathrm{U}}(\sigma, d)=\sum_{g \geq 0} \# P(\sigma,|\sigma|+2 g) d^{-2 g} .
$$

2.4. Monotone factorizations. Consider a permutation $\sigma \in \mathcal{S}_{k}$ and a sequence $f=$ $\left(\tau_{1}, \ldots, \tau_{l}\right)$ of $l$ transpositions satisfying:

- $\tau_{i}=\left(s_{i}, t_{i}\right)$ with $1 \leq s_{i}<t_{i} \leq k$;

- $k \geq t_{1} \geq \cdots \geq t_{l} \geq 1$

- $\sigma=\tau_{1} \cdots \tau_{l}$.

Such a sequence $f$ is called a monotone factorization of length $l$ for $\sigma$. We denote by $\mathcal{F}(\sigma, l)$ the collection of these $f$. Paths in $P(\sigma, l)$ are naturally identified with monotone factorizations.

Lemma 2.8. Let $\sigma \in \mathcal{S}_{k}$. For any nonnegative integer l, there exists a 1-to-1 correspondence between $P(\sigma, l)$ and $\mathcal{F}(\sigma, l)$.

Proof: First, we construct a correspondence $P(\sigma, l) \rightarrow \mathcal{F}(\sigma, l)$. Pick up a path $p=\left(\sigma_{0}, \sigma_{1}, \ldots, \sigma_{k+l}\right)$ in $P(\sigma, l)$. There exist $l$ solid arrows in $p$ :

$$
\sigma_{i_{j}-1} \rightarrow \sigma_{i_{j}}, \quad \text { where } 1 \leq i_{1}<i_{2}<\cdots<i_{l} \leq k+l .
$$

Each solid arrow $\sigma_{i_{j}-1} \rightarrow \sigma_{i_{j}}$ associates with a transposition $\tau_{j}=\left(s_{j}, t_{j}\right)$ satisfying $\sigma_{i_{j}}=\left(s_{j}, t_{j}\right) \sigma_{i_{j}-1}$, where $t_{j}$ is the level of $\sigma_{i_{j}-1}$. Then $p$ gives the relation $e_{k}=$ $\tau_{l} \cdots \tau_{1} \sigma$, or equivalently $\sigma=\tau_{1} \cdots \tau_{l}$. Since levels of $\sigma_{i}$ are weakly decreasing, $t_{j}$ are as well. Thus we have obtained a monotone factorization $f=\left(\tau_{1}, \ldots, \tau_{l}\right)$ in $\mathcal{F}(\sigma, l)$.

Next we construct the inverse map $\mathcal{F}(\sigma, l) \rightarrow P(\sigma, l)$. Let $f=\left(\tau_{1}, \ldots, \tau_{l}\right)$ be a monotone factorization for $\sigma$. Set $\sigma_{0}:=\sigma$ and consider $\tau_{1}=\left(s_{1}, t_{1}\right)$.

- If $t_{1}=k$, then we put $\sigma_{1}:=\tau_{1} \sigma_{0}$ and deal with a solid arrow $\sigma_{0} \rightarrow \sigma_{1}$.

- Assume $t_{1}=k-r$ with $r \geq 1$. Then the monotonicity for $f$ forces $\sigma_{0}(s)=s$ for $k-r+1 \leq s \leq k$, and we can put $\sigma_{i}=\left(\sigma_{i-1}\right)^{\downarrow} \in \mathcal{S}_{k-i}(i=1,2, \ldots, r)$ 
inductively, and finally $\sigma_{r+1}=\tau_{1} \sigma_{r} \in \mathcal{S}_{k-r}$. We thus have obtained a "partial path"

$$
\sigma_{0}-\rightarrow \sigma_{1}-\rightarrow \cdots \rightarrow \sigma_{r} \rightarrow \sigma_{r+1} .
$$

If we repeat this operation for the end point $\sigma_{r+1}(r \geq 0)$ and for the next $\tau_{j}(j=$ $2, \ldots, l)$ until it arrives at $\emptyset$, we can construct a path $p=\left(\sigma_{0}, \ldots, \sigma_{k+l}\right) \in P(\sigma, l)$.

It is clear that the above two correspondences $p \mapsto f$ and $f \mapsto p$ are inverse each other.

Example 2.9. The following objects are identified by the previous lemma.

- A path in $P([4,1,5,3,2], 4)$ :

$$
\begin{aligned}
& {[4,1,5,3,2] \stackrel{(3,5)}{\longrightarrow}[4,1,3,5,2] \stackrel{(2,5)}{\longrightarrow}[4,1,3,2,5] \rightarrow[4,1,3,2]} \\
& \stackrel{(2,4)}{\longrightarrow}[2,1,3,4] \rightarrow[2,1,3] \rightarrow[2,1] \stackrel{(1,2)}{\longrightarrow}[1,2] \rightarrow[1]-\rightarrow \emptyset .
\end{aligned}
$$

- a monotone factorization in $\mathcal{F}([4,1,5,3,2], 4)$ :

$$
f=((3,5),(2,5),(2,4),(1,2)),
$$

or the factorization $[4,1,5,3,2]=(3,5)(2,5)(2,4)(1,2)$

\section{Uniform bounds for unitary Weingarten functions}

3.1. Main results. Our main estimate is as follows:

Theorem 3.1. Let $k$ be a positive integer. For any permutation $\sigma \in \mathcal{S}_{k}$ and nonnegative integer $g$, we have

$$
(k-1)^{g} \# P(\sigma,|\sigma|) \leq \# P(\sigma,|\sigma|+2 g) \leq\left(6 k^{7 / 2}\right)^{g} \# P(\sigma,|\sigma|) .
$$

Theorem 3.2. For any $\sigma \in \mathcal{S}_{k}$ and $d>\sqrt{6} k^{7 / 4}$,

$$
\frac{1}{1-\frac{k-1}{d^{2}}} \leq \frac{(-1)^{|\sigma|} d^{k+|\sigma|} \mathrm{Wg}^{\mathrm{U}}(\sigma, d)}{\# P(\sigma,|\sigma|)} \leq \frac{1}{1-\frac{6 k^{7 / 2}}{d^{2}}} .
$$

In addition, the l.h.s inequality is valid for any $d \geq k$.

Proof: It follows Theorems 2.7 and 3.1 immediately.

\subsection{Comments.}

(1) This bound implies that

$$
d^{k+|\sigma|} \mathrm{Wg}^{\mathrm{U}}(\sigma, d) \rightarrow \operatorname{Moeb}(\sigma)\left(:=(-1)^{|\sigma|} \# P(\sigma,|\sigma|)\right)
$$

in $d \rightarrow \infty$ for any given $\sigma$. This was long known. There was also a need for uniform bounds for theoretical purposes, and has actually already had many applications in QIT. Some weaker bounds have been obtained in Montanaro (2013) and Collins et al. (2013). See also Benaych-Georges (2015).

These two bounds are actually not comparable (one is better than the other depending on the nature of $\sigma$ - specifically, its distance to the identity). The bound that we present in this manuscript is optimal within a polynomial factor, and it improves simultaneously on the two previous bounds. 
(2) We believe (because of the full cycle) that the optimal ratio is $1-\frac{k^{3}}{3 d^{2}}$. Indeed, in the case of the full cycle $Z_{k}$ in $\mathcal{S}_{k}$, we know that

$$
\mathrm{Wg}^{\mathrm{U}}\left(Z_{k}, d\right)=\frac{\operatorname{Cat}(k-1)}{(d-k+1) \ldots(d+k-1)} .
$$

Expanding the denominator shows that $\mathrm{Wg}^{\mathrm{U}}\left(Z_{k}, d\right) \sim \operatorname{Cat}(k-1) d^{-2 k+1}$ as soon as $k^{3} / d^{2} \rightarrow 0$. This would be reminiscent of universality (cf for example Soshnikov, 1999). Indeed, in many occurrences of random matrix theory, the largest eigenvalue of eigenvalues has fluctuations of the order $d^{-2 / 3}$ and they can be analyzed through moments methods with moments that grow as the dimension to the power $3 / 2$. This is exactly the phenomenon that we are witnessing here.

As for us, we just obtained our result for $k^{7 / 2} / d^{2} \rightarrow 0$, however, we believe that $k^{3} / d^{2} \rightarrow 0$ is the right bound, and leave it as an open question.

\subsection{The proof of Theorem 3.1.}

3.3.1. The easy estimate. In order to obtain the left estimate of Theorem 3.1, it is enough to show the inequality

$$
(k-1) \# P(\sigma, l) \leq \# P(\sigma, l+2)
$$

for any $l \geq|\sigma|$. Consider a path $p=\left(\sigma_{0}, \ldots, \sigma_{k+l}\right) \in P(\sigma, l)$ and a transposition $\tau$ of the form $\tau=(i, k)$ with $1 \leq i \leq k-1$. Then the sequence

$$
\tilde{p}=\left(\sigma_{-2}, \sigma_{-1}, \sigma_{0}, \ldots, \sigma_{k+l}\right) \quad \text { with } \sigma_{-2}=\sigma \text { and } \sigma_{-1}=\tau \sigma
$$

is a path from $\sigma$ to $\emptyset$, going through $l+2$ solid edges, i.e., $\tilde{p} \in P(\sigma, l+2)$. The map $(\tau, p) \mapsto \tilde{p}$ is clearly injective. This fact gives the desired inequality.

\subsubsection{Estimates for Catalan numbers.}

Lemma 3.3. For $\sigma \in \mathcal{S}_{k}$ with cycle-type $\mu=\left(\mu_{1}, \mu_{2}, \ldots\right)$,

$$
\# P(\sigma,|\sigma|)=\prod_{i=1}^{\ell(\mu)} \operatorname{Cat}\left(\mu_{i}-1\right),
$$

where $\operatorname{Cat}(n)=\frac{(2 n) !}{(n+1) ! n !}$ is the $n$-th Catalan number.

Proof: It is known that $\# \mathcal{F}(\sigma,|\sigma|)=\prod_{i=1}^{\ell(\mu)} \operatorname{Cat}\left(\mu_{i}-1\right)$, see Matsumoto and Novak (2013, Corollary 2.11). We have the result from Lemma 2.8 immediately.

Lemma 3.4. Let $\sigma \in \mathcal{S}_{k}$, and let $\tau$ be a transposition in $\mathcal{S}_{k}$. Then

$$
\# P(\tau \sigma,|\tau \sigma|) \leq 6 k^{3 / 2} \# P(\sigma,|\sigma|) \text {. }
$$

Proof: Let $\mu=\left(\mu_{1}, \mu_{2}, \ldots\right)$ be the cycle-type of $\sigma$. Then it is well known that the cycle-type of $\tau \sigma$ is obtained from $\mu$ by a cut operation or a join operation. By a cut operation, a part $\mu_{r}$ (greater than 1 ) is decomposed into two parts $(i, j$ ) for some positive integers $i, j$ with $i+j=\mu_{r}$. By a join operation, two parts $\mu_{r}, \mu_{s}$ are combined as $\mu_{r}+\mu_{s}$. Therefore, together with Lemma 3.3, we find that the ratio $\frac{\# P(\tau \sigma,|\tau \sigma|)}{\# P(\sigma,|\sigma|)}$ is bounded by

$$
\max _{r+s+2 \leq k}\left\{\frac{\operatorname{Cat}(r+s+1)}{\operatorname{Cat}(r) \operatorname{Cat}(s)}, \frac{\operatorname{Cat}(r) \operatorname{Cat}(s)}{\operatorname{Cat}(r+s+1)}\right\} .
$$


It is clear that $\frac{\operatorname{Cat}(r) \operatorname{Cat}(s)}{\operatorname{Cat}(r+s+1)} \leq 1$ because of the recurrence formula Cat $(n+1)=$ $\sum_{i+j=n} \operatorname{Cat}(i) \operatorname{Cat}(j)$.

Let us estimate the ratio $\frac{\mathrm{Cat}(r+s+1)}{\operatorname{Cat}(r) \operatorname{Cat}(s)}$. Using the Stirling's formula with precise bounds (Robbins, 1955)

$$
\sqrt{2 \pi} n^{n+1 / 2} e^{-n} \leq n ! \leq e n^{n+1 / 2} e^{-n},
$$

we have inequalities for Catalan numbers

$$
\operatorname{Cat}(n)=\frac{1}{n+1} \frac{(2 n) !}{(n !)^{2}} \leq \frac{1}{n} \frac{e(2 n)^{2 n+1 / 2} e^{-2 n}}{\left(\sqrt{2 \pi} n^{n+1 / 2} e^{-n}\right)^{2}}=\frac{e}{\sqrt{2} \pi} \cdot 4^{n} n^{-3 / 2}
$$

and

$$
\operatorname{Cat}(n) \geq \frac{1}{2 n} \frac{(2 n) !}{(n !)^{2}} \geq \frac{1}{2 n} \frac{\sqrt{2 \pi}(2 n)^{2 n+1 / 2} e^{-2 n}}{\left(e n^{n+1 / 2} e^{-n}\right)^{2}}=\frac{\sqrt{\pi}}{e^{2}} \cdot 4^{n} n^{-3 / 2} .
$$

Therefore we see that

$$
\frac{\operatorname{Cat}(r+s+1)}{\operatorname{Cat}(r) \operatorname{Cat}(s)} \leq \frac{\frac{e}{\sqrt{2} \pi} 4^{r+s+1}(r+s+1)^{-3 / 2}}{\frac{\sqrt{\pi}}{e^{2}} 4^{r} r^{-3 / 2} \cdot \frac{\sqrt{\pi}}{e^{2}} 4^{s} s^{-3 / 2}}=\frac{\sqrt{8} e^{5}}{\pi^{2}}\left(\frac{r s}{r+s+1}\right)^{3 / 2} .
$$

Under the condition $r+s \leq k$, this is clearly bounded by

$$
\frac{\sqrt{8} e^{5}}{\pi^{2}}\left[\left(\frac{r s}{r+s}\right)^{3 / 2}\right]_{r=s=\frac{k}{2}}=\frac{e^{5}}{\sqrt{8} \pi^{2}} k^{3 / 2} .
$$

Here a numerical estimate gives $\frac{e^{5}}{\sqrt{8} \pi^{2}}=5.31 \ldots$.

3.3.3. Deep observations for $P(\sigma, l)$. Let us recall the Weingarten graph $\mathcal{G}^{\mathrm{U}}$ defined in $\S 2.3$. Consider a vertex $\sigma \in \mathcal{S}_{k}$ and a path $p=\left(\sigma_{0}, \ldots, \sigma_{k+l}\right) \in P(\sigma, l)$. The path $p$ goes through exactly $l$ solid edges and $k$ dashed edges. Furthermore, we see that

- If $\sigma_{i} \rightarrow \sigma_{i+1}$, then $\sigma_{i}, \sigma_{i+1}$ have the same level $t$ with some $t \in\{2,3, \ldots, k\}$ and satisfy the relation $\sigma_{i+1}=(s, t) \sigma_{i}$ with some $s \in\{1,2, \ldots, t-1\}$. Moreover, we have $\left|\sigma_{i+1}\right|=\left|\sigma_{i}\right| \pm 1$.

- If $\sigma_{i} \rightarrow \sigma_{i+1}$, then $\sigma_{i+1}=\left(\sigma_{i}\right)^{\downarrow}$ and the level of $\sigma_{i+1}$ is smaller by 1 than that of $\sigma_{i}$. Moreover, $\left|\sigma_{i}\right|=\left|\sigma_{i+1}\right|$.

If $l=|\sigma|$, then $\left|\sigma_{i}\right|>\left|\sigma_{i+1}\right|$ whenever $\sigma_{i} \rightarrow \sigma_{i+1}$.

From now on, we assume $l>|\sigma|$. Then there exist solid edges $\sigma_{i} \rightarrow \sigma_{i+1}$ satisfying $\left|\sigma_{i}\right|<\left|\sigma_{i+1}\right|$. We write $j(p):=j$ if the $(j+1)$-th solid edge in $p$ is the first one among them. Since $|\sigma|<k$ for all $\sigma \in \mathcal{S}_{k}$, the number $j(p)$ should be in $\{0,1,2, \ldots, k-2\}$. Let $\sigma_{j+k-r} \rightarrow \sigma_{j+k-r+1}$ be the present $(j+1)$-th solid edge in $p$. Then, the part $\left(\sigma_{0}, \ldots, \sigma_{j+k-r}\right)$ of $p$ goes through $j$ solid edge and $k-r$ dashed edges, and therefore $\sigma_{j+k-r}$ and $\sigma_{j+k-r+1}$ are of level $r$. Furthermore, the definition of $j=j(p)$ implies that $\left|\sigma_{j+k-r}\right|=|\sigma|-j$ and $\left|\sigma_{j+k-r+1}\right|=\left|\sigma_{j+k-r}\right|+1$.

We now put

$$
P_{j}(\sigma, l)=\{p \in P(\sigma, l) \mid j(p)=j\}
$$

for $j \in\{0,1,2, \ldots, k-2\}$. We have the decomposition

$$
P(\sigma, l)=\bigcup_{j=0}^{k-2} P_{j}(\sigma, l) .
$$


Let $2 \leq r \leq k$, and let $\rho, \rho^{\prime} \in \mathcal{S}_{r}$ be two different permutations in $\mathcal{S}_{r}$ connected by a solid edge. We furthermore put

$$
P_{j}\left(\sigma, \rho, \rho^{\prime}, l\right)=\left\{p=\left(\sigma_{0}, \ldots, \sigma_{k+l}\right) \in P_{j}(\sigma, l) \mid \sigma_{j+k-r}=\rho, \sigma_{j+k-r+1}=\rho^{\prime}\right\} .
$$

As we saw in the previous paragraph, this set is nonempty only if

$$
j=|\sigma|-|\rho| \quad \text { and } \quad\left|\rho^{\prime}\right|=|\rho|+1 .
$$

We have thus obtained the decomposition

$$
P_{j}(\sigma, l)=\bigcup_{r=2}^{k} \bigcup_{\rho \in S_{r}} \bigcup_{\substack{r \\ \rho^{\prime} \in S_{r} \\ \rho \rightarrow \rho^{\prime}}} P_{j}\left(\sigma, \rho, \rho^{\prime}, l\right) .
$$

Let us consider each set $P_{j}\left(\sigma, \rho, \rho^{\prime}, l\right)$ with $l=|\sigma|+2 g$ and suppose that it is nonempty. Decompose each path

$$
p=\left(\sigma_{0}, \ldots, \sigma_{j+k-r}, \sigma_{j+k-r+1}, \ldots, \sigma_{k+l}\right) \in P_{j}\left(\sigma, \rho, \rho^{\prime}, l\right)
$$

into two parts $q=\left(\sigma_{0}, \ldots, \sigma_{j+k-r}\right)$ and $q^{\prime}=\left(\sigma_{j+k-r+1}, \ldots, \sigma_{k+l}\right)$. Then $q$ is a sequence (or a partial path) from $\sigma=\sigma_{0}$ to $\sigma_{j+k-r}=\rho$, going through $j$ solid edges and $k-r$ dashed edges. Also, $q^{\prime}$ is a path from $\rho^{\prime}$ to $\emptyset$ going through $l-j-1$ solid edges and $r$ dashed edges. Since $l-j-1=(|\sigma|+2 g)-(|\sigma|-|\rho|)-1=$ $|\rho|+2 g-1=\left|\rho^{\prime}\right|+2 g-2$ by (3.1), the path $q^{\prime}$ belongs to $P\left(\rho^{\prime},\left|\rho^{\prime}\right|+2 g-2\right)$. We thus obtain the bijection

$$
P_{j}\left(\sigma, \rho, \rho^{\prime},|\sigma|+2 g\right) \cong \tilde{P}_{j}(\sigma, \rho) \times P\left(\rho^{\prime},\left|\rho^{\prime}\right|+2 g-2\right),
$$

where $\tilde{P}_{j}(\sigma, \rho)$ is, by definition, the collection of all partial paths $q=$ $\left(\sigma_{0}, \ldots, \sigma_{j+k-r}\right)$ from $\sigma=\sigma_{0}$ to $\sigma_{j+k-r}=\rho$, going through $j(=|\sigma|-|\rho|)$ solid edges and $k-r$ dashed edges.

3.3.4. Proof of the right estimate in Theorem 3.1. We shall prove inequalities

$$
\# P(\sigma,|\sigma|+2 g) \leq\left(6 k^{7 / 2}\right)^{g} \# P(\sigma,|\sigma|), \quad\left(\sigma \in \mathcal{S}_{k}\right)
$$

by induction on $g$. Note that the case where $g=0$ is trivial. Assume that $g>0$. The induction hypothesis claims that, for all $r \geq 1$ and for all $\eta \in \mathcal{S}_{r}$, it holds that

$$
\# P(\eta,|\eta|+2 g-2) \leq\left(6 r^{7 / 2}\right)^{g-1} \# P(\eta,|\eta|) .
$$

Let $\sigma \in \mathcal{S}_{k}$ and consider $P(\sigma,|\sigma|+2 g)$. The cardinality of each nonempty subset $P_{j}\left(\sigma, \rho, \rho^{\prime},|\sigma|+2 g\right)$ is estimated as follows:

$$
\begin{aligned}
\# P_{j}\left(\sigma, \rho, \rho^{\prime},|\sigma|+2 g\right) & =\# \tilde{P}_{j}(\sigma, \rho) \cdot \# P\left(\rho^{\prime},\left|\rho^{\prime}\right|+2 g-2\right) \\
& \leq \# \tilde{P}_{j}(\sigma, \rho) \cdot\left(6 k^{7 / 2}\right)^{g-1} \# P\left(\rho^{\prime},\left|\rho^{\prime}\right|\right) \\
& \leq \# \tilde{P}_{j}(\sigma, \rho) \cdot\left(6 k^{7 / 2}\right)^{g-1} 6 k^{3 / 2} \cdot \# P(\rho,|\rho|) .
\end{aligned}
$$

Here we have used (3.2), (3.3), and Lemma 3.4 in each step. Together with the fact that, given $\rho \in \mathcal{S}_{r}$, there are $r-1$ possibilities for $\rho^{\prime}$, we obtain

$$
\# \bigcup_{\rho^{\prime}} P_{j}\left(\sigma, \rho, \rho^{\prime},|\sigma|+2 g\right) \leq \# \tilde{P}_{j}(\sigma, \rho) \cdot\left(6 k^{7 / 2}\right)^{g-1} 6 k^{3 / 2} \cdot k \cdot \# P(\rho,|\rho|) .
$$


Here, since the natural map

$$
\begin{aligned}
& \bigcup_{r} \bigcup_{\rho \in \mathcal{S}_{r}}\left(\tilde{P}_{j}(\sigma, \rho) \times P(\rho,|\rho|)\right) \rightarrow P(\sigma,|\sigma|): \\
& \left(\left(\sigma, \sigma_{1}, \ldots, \sigma_{j+k-r-1}, \rho\right),\left(\rho, \sigma_{1}^{\prime}, \ldots, \sigma_{|\rho|+r-1}^{\prime}, \emptyset\right)\right) \\
& \mapsto \underbrace{\sigma, \sigma_{1}, \ldots, \sigma_{j+k-r-1}, \rho, \sigma_{1}^{\prime}, \ldots, \sigma_{|\rho|+r-1}^{\prime}, \emptyset}_{\text {this has }|\sigma| \text { solid edges and } k \text { dashed edges }}),
\end{aligned}
$$

is well-defined and injective, summing over $\rho$ 's, we have

$$
\# P_{j}(\sigma,|\sigma|+2 g) \leq\left(6 k^{7 / 2}\right)^{g-1} 6 k^{5 / 2} \cdot \# P(\sigma,|\sigma|) .
$$

Summing over $j$, one gets

$$
\# P(\sigma,|\sigma|+2 g) \leq\left(6 k^{7 / 2}\right)^{g-1} \cdot 6 k^{5 / 2} \cdot k \cdot \# P(\sigma,|\sigma|)=\left(6 k^{7 / 2}\right)^{g} \# P(\sigma,|\sigma|),
$$

as desired. We have thus completed the proof of Theorem 3.1.

\section{Orthogonal groups}

In this section, we develop the case for orthogonal groups

$$
\mathrm{O}(d)=\left\{g \in \mathrm{GL}(d, \mathbb{C}) \mid g g^{\mathrm{t}}=I_{d}\right\} .
$$

Most parts of the present section is same with the unitary case.

4.1. Weingarten calculus. Suppose that $d, k$ are positive integers with $d \geq 2 k$. Let $\mathcal{P}_{2}(2 k)$ be the set of pair partitions on $\{1,2, \ldots, 2 k\}$. A pair partition $\mathfrak{m} \in \mathcal{P}_{2}(2 k)$ is expressed in the form

$$
\mathfrak{m}=\{\mathfrak{m}(1), \mathfrak{m}(2)\}\{\mathfrak{m}(3), \mathfrak{m}(4)\} \cdots\{\mathfrak{m}(2 k-1), \mathfrak{m}(2 k)\} .
$$

An ordered sequence $\mathbf{i}=\left(i_{1}, \ldots, i_{2 k}\right)$ of $2 k$ positive integers is called admissible for $\mathfrak{m}$ if it holds that

$$
\{r, s\} \in \mathfrak{m} \quad \Rightarrow \quad i_{r}=i_{s} .
$$

Furthermore, $\mathbf{i}$ is called strongly admissible for $\mathfrak{m}$ if it holds that

$$
\{r, s\} \in \mathfrak{m} \quad \Leftrightarrow \quad i_{r}=i_{s} .
$$

For example, if $\mathfrak{m}=\{1,3\}\{2,6\}\{4,5\}$ then $(2,1,2,2,2,1)$ is admissible for $\mathfrak{m}$ but not strongly admissible, and $(2,1,2,3,3,1)$ is strongly admissible.

The symmetric group $\mathcal{S}_{2 k}$ acts transitively on $\mathcal{P}_{2}(2 k)$ by

$$
\sigma \cdot \mathfrak{m}=\{\sigma(\mathfrak{m}(1)), \sigma(\mathfrak{m}(2))\}\{\sigma(\mathfrak{m}(3)), \sigma(\mathfrak{m}(4))\} \cdots\{\sigma(\mathfrak{m}(2 k-1)), \sigma(\mathfrak{m}(2 k))\} .
$$

In particular, we see that

$$
\sigma \cdot \mathfrak{e}=\{\sigma(1), \sigma(2)\} \cdots\{\sigma(2 k-1), \sigma(2 k)\}
$$

for the "trivial pair partition"

$$
\mathfrak{e}=\mathfrak{e}_{k}=\{1,2\}\{3,4\} \cdots\{2 k-1,2 k\} .
$$

For two pair partitions $\mathfrak{m}, \mathfrak{n}$ in $\mathcal{P}_{2}(2 k)$, we let $\mathbf{i}=\left(i_{1}, \ldots, i_{2 k}\right)$ and $\mathbf{j}=\left(j_{1}, \ldots, j_{2 k}\right)$ to be strongly admissible for $\mathfrak{m}$ and $\mathfrak{n}$, respectively. Then the orthogonal Weingarten function $\mathrm{Wg}^{\mathrm{O}}(\mathfrak{m}, \mathfrak{n}, d)$ is defined by

$$
\mathrm{Wg}^{\mathrm{O}}(\mathfrak{m}, \mathfrak{n}, d)=\int_{\mathrm{O}(d)} u_{i_{1} j_{1}} u_{i_{2} j_{2}} \cdots u_{i_{2 k} j_{2 k}} d \mu .
$$


Here $d \mu=d \mu^{\mathrm{O}(d)}$ denotes the normalized Haar measure on $\mathrm{O}(d)$. For example, if $\mathfrak{m}=\{1,3\}\{2,6\}\{4,5\}$ and $\mathfrak{n}=\{1,2\}\{3,4\}\{5,6\}$, then we can write

$$
\mathrm{Wg}^{\mathrm{O}}(\mathfrak{m}, \mathfrak{n}, d)=\int_{\mathrm{O}(d)} u_{21} u_{11} u_{22} u_{32} u_{33} u_{13} d \mu .
$$

By virtue of the bi-invariant property of the Haar measure, this definition is independent of choices of strongly admissible sequences.

The Weingarten calculus for orthogonal groups is stated as follows.

Lemma 4.1 (Collins and Śniady, 2006). For two sequences

$$
\mathbf{i}=\left(i_{1}, \ldots, i_{2 k}\right), \quad \mathbf{j}=\left(j_{1}, \ldots, j_{2 k}\right)
$$

of positive integers in $\{1,2, \ldots, d\}$, we have

$$
\begin{aligned}
& \int_{\mathrm{O}(d)} u_{i_{1} j_{1}} u_{i_{2} j_{2}} \cdots u_{i_{2 k} j_{2 k}} d \mu \\
& =\sum_{\mathfrak{m} \in \mathcal{P}_{2}(2 k)} \sum_{\mathfrak{n} \in \mathcal{P}_{2}(2 k)} \Delta_{\mathfrak{m}}(\mathbf{i}) \Delta_{\mathfrak{n}}(\mathbf{j}) \mathrm{Wg}^{\mathrm{O}}(\mathfrak{m}, \mathfrak{n}, d) .
\end{aligned}
$$

Here $\Delta_{\mathfrak{m}}(\mathbf{i})$ is defined by

$$
\Delta_{\mathfrak{m}}(\mathbf{i})= \begin{cases}1 & \text { if } \mathbf{i} \text { is admissible for } \mathfrak{m}, \\ 0 & \text { otherwise. }\end{cases}
$$

We will use the following lemma later.

Lemma 4.2. For any $\sigma \in \mathcal{S}_{2 k}$ and $\mathfrak{m}, \mathfrak{n} \in \mathcal{P}_{2}(2 k)$, we have

$$
\mathrm{Wg}^{\mathrm{O}}(\sigma \cdot \mathfrak{m}, \sigma \cdot \mathfrak{n}, d)=\mathrm{Wg}^{\mathrm{O}}(\mathfrak{m}, \mathfrak{n}, d)
$$

Proof: It is easy to see that: if $\mathbf{i}$ is a strongly admissible sequence for $\mathfrak{m}$, then $\mathbf{i}^{\sigma}=\left(i_{\sigma(1)}, \ldots, i_{\sigma(2 k)}\right)$ is strongly admissible for $\sigma \cdot \mathfrak{m}$. Therefore

$$
\begin{aligned}
& \mathrm{Wg}^{\mathrm{O}}(\sigma \cdot \mathfrak{m}, \sigma \cdot \mathfrak{n}, d)=\int_{\mathrm{O}(d)} u_{i_{\sigma(1)}, j_{\sigma(1)}} \cdots u_{i_{\sigma(2 k)}, j_{\sigma(2 k)}} d \mu \\
= & \int_{\mathrm{O}(d)} u_{i_{1}, j_{1}} \cdots u_{i_{2 k}, j_{2 k}} d \mu=\mathrm{Wg}^{\mathrm{O}}(\mathfrak{m}, \mathfrak{n}, d) .
\end{aligned}
$$

We write $\mathrm{Wg}^{\mathrm{O}}(\mathfrak{e}, \mathfrak{m}, d)$ by $\mathrm{Wg}^{\mathrm{O}}(\mathfrak{m}, d)$, which is also called the orthogonal Weingarten function. For each $\mathfrak{m}$, there exist some $\sigma_{\mathfrak{m}} \in \mathcal{S}_{2 k}$ with $\sigma_{\mathfrak{m}} \cdot \mathfrak{e}=\mathfrak{m}$, and

$$
\mathrm{Wg}^{\mathrm{O}}(\mathfrak{m}, \mathfrak{n}, d)=\mathrm{Wg}^{\mathrm{O}}\left(\sigma_{\mathfrak{m}} \cdot \mathfrak{e}, \mathfrak{n}, d\right)=\mathrm{Wg}^{\mathrm{O}}\left(\mathfrak{e}, \sigma_{\mathfrak{m}}^{-1} \cdot \mathfrak{n}, d\right)=\mathrm{Wg}^{\mathrm{O}}\left(\sigma_{\mathfrak{m}}^{-1} \cdot \mathfrak{n}, d\right)
$$

by Lemma 4.2. Thus, it is enough to deal with the family $\left\{\mathrm{Wg}^{\mathrm{O}}(\mathfrak{m}, d)\right\}_{\mathfrak{m} \in \mathcal{P}_{2}(2 k)}$ on behalf of $\left\{\mathrm{Wg}^{\mathrm{O}}(\mathfrak{m}, \mathfrak{n}, d)\right\}_{\mathfrak{m}, \mathfrak{n} \in \mathcal{P}_{2}(2 k)}$.

\subsection{Orthogonality relations.}

Lemma 4.3. The orthogonal Weingarten function satisfies the following formula: For each $\mathfrak{m} \in \mathcal{P}_{2}(2 k)$,

$$
d \mathrm{Wg}^{\mathrm{O}}(\mathfrak{m}, d)=-\sum_{i=1}^{2 k-2} \mathrm{Wg}^{\mathrm{O}}((i, 2 k-1) \cdot \mathfrak{m}, d)+\delta_{\{2 k-1,2 k\} \in \mathfrak{m}} \mathrm{Wg}^{\mathrm{O}}\left(\mathfrak{m}^{\downarrow}, d\right) .
$$

Here 
- $\delta_{\{2 k-1,2 k\} \in \mathfrak{m}}= \begin{cases}1 & \text { if }\{2 k-1,2 k\} \in \mathfrak{m}, \\ 0 & \text { otherwise. }\end{cases}$

- $\mathfrak{m} \downarrow$ is the pair partition in $\mathcal{P}_{2}(2 k-2)$ obtained from $\mathfrak{m}$ by removing the block $\{2 k-1,2 k\}$ (if possible).

Proof: In the present proof, we abbreviate as $\mathrm{Wg}(\mathfrak{m}, \mathfrak{n})=\mathrm{Wg}^{\mathrm{O}}(\mathfrak{m}, \mathfrak{n}, d)$ and $\mathrm{Wg}(\mathfrak{m})$ $=\mathrm{Wg}^{\mathrm{O}}(\mathfrak{m}, d)$. Let $\mathbf{i}=\left(i_{1}, \ldots, i_{2 k}\right)$ be a strongly admissible sequence for $\mathfrak{m}$. Consider the sum of integrals

$$
\sum_{i=1}^{d} \int_{\mathrm{O}(d)}\left(\prod_{r=1}^{k-1} u_{i_{2 r-1}, r} u_{i_{2 r}, r}\right) \cdot u_{i_{2 k-1}, i} u_{i_{2 k}, i} d \mu .
$$

Since $U=\left(u_{i j}\right)$ is orthogonal, we have $\sum_{i=1}^{d} u_{i_{2 k-1}, i} u_{i_{2 k}, i}=\delta_{i_{2 k-1}, i_{2 k}}$, which is equal to $\delta_{\{2 k-1,2 k\} \in \mathfrak{m}}$. Note that, if $\{2 k-1,2 k\} \in \mathfrak{m}$, the sequence $\left(i_{1}, \ldots, i_{2 k-2}\right)$ is strongly admissible for $\mathfrak{m}^{\downarrow}$. Therefore (4.2) equals

$$
\delta_{\{2 k-1,2 k\} \in \mathfrak{m}} \int_{\mathrm{O}(d)}\left(\prod_{r=1}^{k-1} u_{i_{2 r-1}, r} u_{i_{2 r}, r}\right) d \mu=\delta_{\{2 k-1,2 k\} \in \mathfrak{m}} \mathrm{Wg}\left(\mathfrak{m}^{\downarrow}\right) .
$$

On the other hand, the sequence $\mathbf{j}=(1,1,2,2, \ldots, k-1, k-1, i, i)$ is admissible for $\mathfrak{e}$. Moreover, if $i<k$, the sequence $\mathbf{j}$ is admissible for $(2 i-1,2 k-1) \cdot \mathfrak{e}$ and $(2 i, 2 k-1) \cdot e$. Hence, it follows from Lemma 4.1 that:

- if $i \geq k$, then

$$
\int_{\mathrm{O}(d)}\left(\prod_{r=1}^{k-1} u_{i_{2 r-1}, r} u_{i_{2 r}, r}\right) \cdot u_{i_{2 k-1}, i} u_{i_{2 k}, i} d \mu=\mathrm{Wg}(\mathfrak{m}, \mathfrak{e})=\mathrm{Wg}(\mathfrak{m}) ;
$$

- if $i<k$, then

$$
\begin{aligned}
& \int_{\mathrm{O}(d)}\left(\prod_{r=1}^{k-1} u_{i_{2 r-1}, r} u_{i_{2 r}, r}\right) \cdot u_{i_{2 k-1}, i} u_{i_{2 k}, i} d \mu \\
& =\mathrm{Wg}(\mathfrak{m}, \mathfrak{e})+\mathrm{Wg}(\mathfrak{m},(2 i-1,2 k-1) \cdot \mathfrak{e})+\mathrm{Wg}(\mathfrak{m},(2 i, 2 k-1) \cdot \mathfrak{e}) \\
& =\mathrm{Wg}(\mathfrak{m})+\mathrm{Wg}((2 i-1,2 k-1) \cdot \mathfrak{m})+\mathrm{Wg}((2 i, 2 k-1) \cdot \mathfrak{m}) .
\end{aligned}
$$

Summing up over $i$, the equation (4.2) equals

$$
d \mathrm{Wg}(\mathfrak{m})+\sum_{j=1}^{2 k-2} \mathrm{Wg}((j, 2 k-1) \cdot \mathfrak{m}) .
$$

Example 4.4. We again abbreviate as $\mathrm{Wg}(\mathfrak{m})=\mathrm{Wg}^{\mathrm{O}}(\mathfrak{m}, d)$. Equation (4.1) with $k=2$ gives three identities

$$
\begin{aligned}
& d \mathrm{Wg}(\{1,2\}\{3,4\})=-\mathrm{Wg}(\{1,3\}\{2,4\})-\mathrm{Wg}(\{1,4\}\{2,3\})+\mathrm{Wg}(\{1,2\}) ; \\
& d \mathrm{Wg}(\{1,3\}\{2,4\})=-\mathrm{Wg}(\{1,2\}\{3,4\})-\mathrm{Wg}(\{1,3\}\{2,4\}) \\
& d \mathrm{Wg}(\{2,3\}\{1,4\})=-\operatorname{Wg}(\{2,3\}\{1,4\})-\mathrm{Wg}(\{1,2\}\{3,4\}) .
\end{aligned}
$$

The second and third identities imply that

$$
\mathrm{Wg}(\{1,3\}\{2,4\})=\mathrm{Wg}(\{2,3\}\{1,4\})=-\frac{1}{d+1} \mathrm{Wg}(\{1,2\}\{3,4\}) .
$$


Furthermore, the first identity gives

so that

$$
d \mathrm{Wg}(\{1,2\}\{3,4\})=\frac{2}{d+1} \mathrm{Wg}(\{1,2\}\{3,4\})+\mathrm{Wg}(\{1,2\}),
$$

$$
\mathrm{Wg}(\{1,2\}\{3,4\})=\frac{d+1}{(d+2)(d-1)} \cdot \mathrm{Wg}(\{1,2\})=\frac{d+1}{(d+2) d(d-1)} .
$$

4.3. Weingarten graphs. The Weingarten graph for the orthogonal group can be defined quite similar way to the unitary group. We only mention the difference between them. We consider the graph $\mathcal{G}^{\mathrm{O}}=(V, E)$. The vertex set $V$ is $\bigsqcup_{k=0}^{\infty} \mathcal{P}_{2}(2 k)$. For convenience, we set $\mathcal{P}_{2}(0)=\{\emptyset\}$ with the 'empty pair partition' $\emptyset=\mathfrak{e}_{0}$. Two vertices $\mathfrak{m}, \mathfrak{n}$ of level $k$ (i.e. in $\mathcal{P}_{2}(2 k)$ ) are connected by a solid arrow as $\mathfrak{m} \rightarrow \mathfrak{n}$ if and only if

$$
\mathfrak{n}=(i, 2 k-1) \cdot \mathfrak{m} \quad \text { with some } i \text { smaller than } 2 k-1 .
$$

For each $k \geq 1$, a vertex $\mathfrak{m}$ of level $k$ and a vertex $\mathfrak{m}^{\prime}$ of level $k-1$ are connected by a dashed arrow as $\mathfrak{m} \rightarrow \mathfrak{m}^{\prime}$ if and only if $\{2 k-1,2 k\} \in \mathfrak{m}$ and $\mathfrak{m}^{\downarrow}=\mathfrak{m}^{\prime}$.

We also consider a path $p=\left(\mathfrak{m}_{0}, \mathfrak{m}_{1}, \ldots, \mathfrak{m}_{l+k}\right)$ as in the unitary case. Denote by $P(\mathfrak{m}, l)$ the collection of all paths from $\mathfrak{m} \in \mathcal{P}_{2}(2 k)$ to $\emptyset$, going through $l$ solid edges and $k$ dashed edges.

Lemma 4.5. For $\mathfrak{m} \in \mathcal{P}_{2}(2 k)$, we have the formal expansion

$$
\mathrm{Wg}^{\mathrm{O}}(\mathfrak{m}, d)=d^{-k} \sum_{l \geq 0} \# P(\mathfrak{m}, l)\left(-d^{-1}\right)^{l} .
$$

Proof: It is same with the proof of Lemma 2.5. Use Lemma 4.3.

For each pair partition $\mathfrak{m} \in \mathcal{P}_{2}(2 k)$, we associate with the coset-type $\mu$, which is an integer partition of $k$. We can see its definition in e.g. Matsumoto $(2011,2013)$. Put $|\mathfrak{m}|=k-\ell(\mu)$. For example, $|\mathfrak{m}|=0$ if and only if $\mathfrak{m}=\mathfrak{e}_{k}$.

We can observe the fact $\left|\mathfrak{m}^{\downarrow}\right|=|\mathfrak{m}|$ and

$$
|\tau \cdot \mathfrak{m}|-|\mathfrak{m}| \in\{-1,0,1\}
$$

for any transposition $\tau$ in $\mathcal{S}_{2 k}$. We emphasis that the equality $|\tau \cdot \mathfrak{m}|=|\mathfrak{m}|$ may happen, different from the unitary case. We have obtained the following expansion. Note that, different from the unitary case, the summation of the right hand side is alternating.

Theorem 4.6. For each $\mathfrak{m} \in \mathcal{P}_{2}(2 k)$, we have the formal expansion

$$
(-1)^{|\mathfrak{m}|} d^{|\mathfrak{m}|+k} \mathrm{Wg}^{\mathrm{O}}(\mathfrak{m}, d)=\sum_{g \geq 0} \# P(\mathfrak{m},|\mathfrak{m}|+g)(-d)^{-g} .
$$

4.4. Monotone factorizations. Consider a pair partition $\mathfrak{m} \in \mathcal{P}_{2}(2 k)$ and a sequence $f=\left(\tau_{1}, \ldots, \tau_{l}\right)$ of $l$ transpositions satisfying:

- $\tau_{i}=\left(s_{i}, 2 t_{i}-1\right)$ with $1 \leq s_{i}<2 t_{i}-1 \leq 2 k-1$;

- $k \geq t_{1} \geq t_{2} \geq \cdots \geq t_{l} \geq 1$

- $\mathfrak{m}=\left(\tau_{1} \cdots \tau_{l}\right) \cdot \mathfrak{e}$.

Such a sequence $f$ is called a monotone factorization of length $l$ for $\mathfrak{m}$. We denote by $\mathcal{F}(\mathfrak{m}, l)$ the collection of these $f$. 
Lemma 4.7. Let $\mathfrak{m} \in \mathcal{P}_{2}(2 k)$. For any nonnegative integer $l$, there exists a 1-to-1 correspondence between $P(\mathfrak{m}, l)$ and $\mathcal{F}(\mathfrak{m}, l)$.

Proof: It is the same with that of Lemma 2.8.

4.5. Symplectic groups. Consider the symplectic group

$$
\mathrm{Sp}(d)=\{g \in \mathrm{U}(2 d) \mid g J=J g\}, \quad \text { with } \quad J=J_{d}=\left(\begin{array}{cc}
O_{d} & I_{d} \\
-I_{d} & O_{d}
\end{array}\right) .
$$

The Weingarten calculus for $\operatorname{Sp}(d)$ can be described in a similar way to orthogonal groups $\mathrm{O}(d)$. We do not state the specific formula here and we are interested in only the absolute value of the Weingarten function. The readers can see the exact formula in Collins and Stolz (2008); Matsumoto (2013). For each pair partition $\mathfrak{m} \in \mathcal{P}_{2}(2 k)$, the symplectic Weingarten function $\mathrm{Wg}^{\mathrm{Sp}}(\mathfrak{m}, d)$ is given by

$$
\pm \mathrm{Wg}^{\mathrm{Sp}}(\mathfrak{m}, d)=\mathrm{Wg}^{\mathrm{O}}(\mathfrak{m},-2 d),
$$

up to sign. Here the quantity $\mathrm{Wg}^{\mathrm{O}}(\mathfrak{m},-2 d)$ is obtained from the orthogonal Weingarten function $\mathrm{Wg}^{\mathrm{O}}(\mathfrak{m}, d)$ by replacing $d$ with $-2 d$ formally. Therefore, from Theorem 4.6, we can expand it as follows.

Theorem 4.8. For each $\mathfrak{m} \in \mathcal{P}_{2}(2 k)$, we have the formal expansion

$$
(2 d)^{|\mathfrak{m}|+k}\left|\mathrm{Wg}^{\mathrm{Sp}}(\mathfrak{m}, d)\right|=\sum_{g \geq 0} \# P(\mathfrak{m},|\mathfrak{m}|+g)(2 d)^{-g} .
$$

4.6. Uniform bounds.

Theorem 4.9. Let $k$ be a positive integer. For any pair partition $\mathfrak{m} \in \mathcal{P}_{2}(2 k)$ and nonnegative integer $g$, we have

$$
\begin{aligned}
& \# P(\sigma,|\mathfrak{m}|+2 g) \geq(2 k-2)^{g} \# P(\mathfrak{m},|\mathfrak{m}|), \\
& \# P(\sigma,|\mathfrak{m}|+g) \leq\left(12 k^{7 / 2}\right)^{g} \# P(\mathfrak{m},|\mathfrak{m}|) .
\end{aligned}
$$

Theorem 4.10. For any $\mathfrak{m} \in \mathcal{P}_{2}(2 k)$ and $d>6 k^{7 / 2}$,

$$
\frac{\# P(\mathfrak{m},|\mathfrak{m}|)}{1-\frac{k-1}{2 d^{2}}} \leq(2 d)^{|\mathfrak{m}|+k}\left|\mathrm{Wg}^{\mathrm{Sp}}(\mathfrak{m}, d)\right| \leq \frac{\# P(\mathfrak{m},|\mathfrak{m}|)}{1-\frac{6 k^{7 / 2}}{d}} .
$$

Proof: This is a direct consequence from Theorems 4.8 and 4.9. The left estimate is obtained by ignoring odd degree terms:

$$
(2 d)^{|\mathfrak{m}|+k}\left|\mathrm{Wg}^{\mathrm{Sp}}(\mathfrak{m}, d)\right| \geq(2 d)^{|\mathfrak{m}|+k} \sum_{g \geq 0} \# P(\mathfrak{m},|\mathfrak{m}|+2 g)(2 d)^{-2 g} .
$$

Since the orthogonal Weingarten function is an alternating sum (Theorem 4.6), we obtain a slightly weaker upper bound. We can also obtain a lower bound, but due to the fact that the orthogonal case involves signed sums, it is not as sharp as in the unitary or symplectic case.

Theorem 4.11. For any $\mathfrak{m} \in \mathcal{P}_{2}(2 k)$ and $d>12 k^{7 / 2}$,

$$
\# P(\mathfrak{m},|\mathfrak{m}|) \frac{1-\frac{24 k^{7 / 2}}{d}}{1-\frac{144 k^{7}}{d^{2}}} \leq(-1)^{|\mathfrak{m}|} d^{|\mathfrak{m}|+k} \mathrm{Wg}^{\mathrm{O}}(\mathfrak{m}, d) \leq \frac{\# P(\mathfrak{m},|\mathfrak{m}|)}{1-\frac{144 k^{7}}{d^{2}}} .
$$


Proof: From Theorem 4.6 we see that the positive value $(-1)^{|\mathfrak{m}|} d^{|\mathfrak{m}|+k} \mathrm{Wg}^{\mathrm{O}}(\mathfrak{m}, d)$ is equal to

$$
\sum_{g \geq 0} \# P(\mathfrak{m},|\mathfrak{m}|+2 g) d^{-2 g}-\sum_{g \geq 0} \# P(\mathfrak{m},|\mathfrak{m}|+2 g+1) d^{-(2 g+1)} .
$$

Applying (4.4) to the first summand and ignoring the second summand, we have

$$
(-1)^{|\mathfrak{m}|} d^{|\mathfrak{m}|+k} \mathrm{Wg}^{\mathrm{O}}(\mathfrak{m}, d) \leq \# P(\mathfrak{m},|\mathfrak{m}|) \sum_{g \geq 0} c^{2 g}=\frac{\# P(\mathfrak{m},|\mathfrak{m}|)}{1-c^{2}}
$$

with $c=12 k^{7 / 2} d^{-1}(<1)$. On the other hand, applying (4.4) to the second summand and ignoring the first summand except the first term, we have

$$
\begin{aligned}
(-1)^{|\mathfrak{m}|} d^{|\mathfrak{m}|+k} \mathrm{Wg}^{\mathrm{O}}(\mathfrak{m}, d) & \geq \# P(\mathfrak{m},|\mathfrak{m}|)-\# P(\mathfrak{m},|\mathfrak{m}|) \sum_{g \geq 0} c^{2 g+1} \\
& =\# P(\mathfrak{m},|\mathfrak{m}|)\left(1-\frac{c}{1-c^{2}}\right) \geq \# P(\mathfrak{m},|\mathfrak{m}|) \cdot \frac{1-2 c}{1-c^{2}} .
\end{aligned}
$$

4.7. Proof of Theorem 4.9. The proof is obtained in a similar way to subsection 3.3. We only mention the difference between them. Note that the first inequality in Theorem 4.9 can be obtained in a similar way to the unitary case. In fact, we can choose $2 k-2$ solid edges connected with $\mathfrak{m} \in \mathcal{P}_{2}(2 k)$.

Let us show the second inequality of the theorem. First we observe the explicit value for $P(\mathfrak{m},|\mathfrak{m}|)$. Recall that the cardinality of $P(\mathfrak{m}, l)$ for the graph $\mathcal{G}^{\mathrm{O}}$ is different from that of $P(\sigma, l)$ for the graph $\mathcal{G}^{\mathrm{U}}$ in general. Nevertheless, the numbers of shortest paths in each case coincide.

Lemma 4.12. For $\mathfrak{m} \in \mathcal{P}_{2}(2 k)$ with coset-type $\mu=\left(\mu_{1}, \mu_{2}, \ldots\right)$,

$$
\# P(\mathfrak{m},|\mathfrak{m}|)=\prod_{i=1}^{\ell(\mu)} \operatorname{Cat}\left(\mu_{i}-1\right) .
$$

Therefore, for any transposition $\tau$ in $\mathcal{S}_{2 k}$, we have

$$
\# P(\tau \cdot \mathfrak{m},|\tau \cdot \mathfrak{m}|) \leq 6 k^{3 / 2} \# P(\mathfrak{m},|\mathfrak{m}|) .
$$

Proof: The first statement is seen in Matsumoto (2011, Theorem 5.4). We also use Lemma 4.7. The latter statement is shown in Lemma 3.4.

Recall the Weingarten graph $\mathcal{G}^{\mathrm{O}}$. Consider a vertex $\mathfrak{m} \in \mathcal{P}_{2}(2 k)$ and a path $p=\left(\mathfrak{m}_{0}, \ldots, \mathfrak{m}_{k+l}\right) \in P(\mathfrak{m}, l)$. Suppose that $l>|\mathfrak{m}|$ and that two vertices $\mathfrak{m}_{i}, \mathfrak{m}_{i+1}$ in $p$ are connected by a solid arrow: $\mathfrak{m}_{i} \rightarrow \mathfrak{m}_{i+1}$. Different from the unitary case, it happens that $\left|\mathfrak{m}_{i+1}\right|-\left|\mathfrak{m}_{i}\right|=-1,0$, or +1 . We write $j(p)=j$ if the $(j+1)$-th solid edge $\mathfrak{m}_{j+k-r} \rightarrow \mathfrak{m}_{j+k-r+1}$ in $p$ (with some $r$ ) is the first solid edge satisfying

$$
\left|\mathfrak{m}_{i}\right| \leq\left|\mathfrak{m}_{i+1}\right| .
$$

The number $j(p)$ is well defined in $\{0,1, \ldots, k-2\}$. Then, the part $\left(\mathfrak{m}_{0}, \ldots, \mathfrak{m}_{j+k-r}\right)$ of $p$ goes through $j$ solid edges and $k-r$ dashed edges, and therefore $\mathfrak{m}_{j+k-r}$ and $\mathfrak{m}_{j+k-r+1}$ are of levels $r$. Furthermore, $\left|\mathfrak{m}_{j+k-r}\right|=|\mathfrak{m}|-j$, and

$$
\left|\mathfrak{m}_{j+k-r+1}\right|=\left|\mathfrak{m}_{j+k-r}\right| \quad \text { or } \quad\left|\mathfrak{m}_{j+k-r+1}\right|=\left|\mathfrak{m}_{j+k-r}\right|+1 .
$$


Like the unitary case, we define

$$
P_{j}(\mathfrak{m}, l)=\{p \in P(\mathfrak{m}, l) \mid j(p)=j\}
$$

and

$$
P_{j}\left(\mathfrak{m}, \mathfrak{n}, \mathfrak{n}^{\prime}, l\right)=\left\{p=\left(\mathfrak{m}_{0}, \ldots, \mathfrak{m}_{k+l}\right) \in P_{j}(\mathfrak{m}, l) \mid \mathfrak{m}_{j+k-r}=\mathfrak{n}, \mathfrak{m}_{j+k-r+1}=\mathfrak{n}^{\prime}\right\}
$$

for pair partitions $\mathfrak{n}, \mathfrak{n}^{\prime}$. This is nonempty only if

- $j=|\mathfrak{m}|-|\mathfrak{n}|$;

- $\mathfrak{n}$ and $\mathfrak{n}^{\prime}$ have the same level $r$ and are connected by a solid edge;

- $\left|\mathfrak{n}^{\prime}\right|=|\mathfrak{n}|$ or $\left|\mathfrak{n}^{\prime}\right|=|\mathfrak{n}|+1$,

which should be compared with (3.1).

Let $g \geq 1$. We can obtain bijections

$$
\begin{aligned}
& P_{j}\left(\mathfrak{m}, \mathfrak{n}, \mathfrak{n}^{\prime},|\mathfrak{m}|+g\right) \\
& \cong \begin{cases}\tilde{P}_{j}(\mathfrak{m}, \mathfrak{n}) \times P\left(\mathfrak{n}^{\prime},\left|\mathfrak{n}^{\prime}\right|+g-2\right) & \text { if }\left|\mathfrak{n}^{\prime}\right|=|\mathfrak{n}|+1, \\
\tilde{P}_{j}(\mathfrak{m}, \mathfrak{n}) \times P\left(\mathfrak{n}^{\prime},\left|\mathfrak{n}^{\prime}\right|+g-1\right) & \text { if }\left|\mathfrak{n}^{\prime}\right|=|\mathfrak{n}|,\end{cases}
\end{aligned}
$$

where $\tilde{P}_{j}(\mathfrak{m}, \mathfrak{n})$ is, by definition, the collection of all partial paths $q=$ $\left(\mathfrak{m}_{0}, \ldots, \mathfrak{m}_{j+k-r}\right)$ from $\mathfrak{m}=\mathfrak{m}_{0}$ to $\mathfrak{m}_{j+k-r}=\mathfrak{n}$, going through $j(=|\mathfrak{m}|-|\mathfrak{n}|)$ solid edges and $k-r$ dashed edges.

We shall show the second inequality in Theorem 4.9 by induction on $g$. Using the induction assumption, a similar discussion to the unitary case gives

$$
\# P_{j}\left(\mathfrak{m}, \mathfrak{n}, \mathfrak{n}^{\prime},|\mathfrak{m}|+g\right) \leq \# \tilde{P}_{j}(\mathfrak{m}, \mathfrak{n}) \cdot\left(12 k^{7 / 2}\right)^{g-1} \cdot 6 k^{3 / 2} \cdot \# P(\mathfrak{n},|\mathfrak{n}|) .
$$

Together with the fact that, given $\mathfrak{n} \in \mathcal{P}_{2}(2 r)$, there are $2 r-2$ possibilities for $\mathfrak{n}^{\prime}$ (since $\mathfrak{n}, \mathfrak{n}^{\prime}$ are connected by a solid edge), we obtain

$$
\# \bigcup_{\mathfrak{n}^{\prime}} P_{j}\left(\mathfrak{m}, \mathfrak{n}, \mathfrak{n}^{\prime},|\mathfrak{m}|+g\right) \leq \# \tilde{P}_{j}(\mathfrak{m}, \mathfrak{n}) \cdot\left(12 k^{7 / 2}\right)^{g-1} \cdot 6 k^{3 / 2} \cdot 2 k \cdot \# P(\mathfrak{n},|\mathfrak{n}|) .
$$

The remaining discussion is same with the unitary case again.

4.8. Discussion for right estimates. In the left estimate of (4.5), we ignored the odd-degree terms. If ones want to find a sharper estimate, we need to compare $\# P(\mathfrak{m},|\mathfrak{m}|+1)$ with $\# P(\mathfrak{m},|\mathfrak{m}|)$. In the present short subsection, we observe the difficulty of a direct comparison.

Let us recall an analogue of Lemma 4.12 for $\# P(\mathfrak{m},|\mathfrak{m}|+1)$. If $I=\left(i_{1}, \ldots, i_{r}\right)$ is a sequence of nonnegative integers, let us define $\mathcal{D}_{I}$ as the set of Dyck paths of length $|I|:=i_{1}+\cdots+i_{r}$ whose height after $i_{1}, i_{1}+i_{2}, \ldots$ steps is zero. For each Dyck path $c \in \mathcal{D}_{I}$, we denote by $\mathcal{A}(c)$ the area under $c$. For example, for the Dyck path $c=(+1,-1,+1,-1) \in \mathcal{D}_{(2,2)}$, the area is $\mathcal{A}(c)=2$, which is a sum of two triangles.

Lemma 4.13 (partially conjectured in Matsumoto, 2011 and proved in Féray, 2012). If $\mu=\left(\mu_{1}, \ldots, \mu_{l}\right)$ is the coset-type of $\mathfrak{m} \in \mathcal{P}_{2}(2 k)$, then we have the expression

$$
\# P(\mathfrak{m},|\mathfrak{m}|+1)=\sum_{c \in \mathcal{D}_{I_{\mu}}} \mathcal{A}(c)=\sum_{i=1}^{\ell}\left(\sum_{c \in \mathcal{D}_{\left(\mu_{i}-1\right)}} \mathcal{A}(c)\right) \prod_{j \neq i} \operatorname{Cat}\left(\mu_{j}-1\right)
$$

with $I_{\mu}=\left(\mu_{1}-1, \ldots, \mu_{l}-1\right)$. 
Lemma 4.12 states the formula $\# P(\mathfrak{m},|\mathfrak{m}|)=\left|\mathcal{D}_{I_{\mu}}\right|=\sum_{c \in \mathcal{D}_{I_{\mu}}}$ 1. If $\mu \neq\left(1^{k}\right)$, then we obtain a trivial inequality

$$
\begin{aligned}
\# P(\mathfrak{m},|\mathfrak{m}|+1) & \geq\left(\sum_{c \in \mathcal{D}_{\left(\mu_{1}-1\right)}} \mathcal{A}(c)\right) \prod_{j \geq 2} \operatorname{Cat}\left(\mu_{j}-1\right) \\
& \geq \prod_{j \geq 1} \operatorname{Cat}\left(\mu_{j}-1\right) \\
& =\# P(\mathfrak{m},|\mathfrak{m}|) .
\end{aligned}
$$

However, if $\mu=\left(1^{k}\right)$, then

$$
\# P(\mathfrak{m},|\mathfrak{m}|+1)=0 \quad \text { and } \quad \# P(\mathfrak{m},|\mathfrak{m}|)=1 .
$$

Thus, it is not clear to find a uniform estimate between $\# P(\mathfrak{m},|\mathfrak{m}|+1)$ and $\# P(\mathfrak{m},|\mathfrak{m}|)$.

\section{Compact symmetric spaces}

Let $G / K$ be a classical compact symmetric space. We may assume that $G$ is a compact matrix group and $K$ is a closed subgroup fixed by a so-called Cartan involution $\theta$ of $G$. Then the space $G / K$ is identified with the subset $\mathbb{S}=\left\{g \theta(g)^{-1} \mid g \in G\right\}$ of $G$. The group $G$ acts on $\mathbb{S}$ by $g . s=g s \theta(g)^{-1}(g \in G, s \in \mathbb{S})$. It is known that there exists the unique probability measure $d \nu$ on $\mathbb{S}$, which is invariant under this action. Cartan (1927) classified classical compact symmetric spaces into seven series, which are labelled as A I, A II, A III, BD I, C I, C II, and D III.

The Weingarten calculus of $G / K$ is the method for computations of integrals of the forms

$$
\int_{\mathbb{S}} s_{i_{1} j_{1}} \cdots s_{i_{k} j_{k}} d \nu \quad \text { or } \quad \int_{\mathbb{S}} s_{i_{1} j_{1}} \cdots s_{i_{k} j_{k}} \frac{s_{i_{1}^{\prime} j_{1}^{\prime}} \cdots s_{i_{l}^{\prime} j_{l}^{\prime}}}{d \nu}
$$

where $s_{i j}: \mathbb{S} \rightarrow \mathbb{C}$ is the $i j$-coordinate function. This is arose in Collins and Stolz (2008) and much developed in Matsumoto (2013) by applying harmonic analysis of symmetric groups.

In this section, we focus on two symmetric spaces of types A I and A III. As we did for unitary groups and orthogonal groups, we will find orthogonal relations for Weingarten functions of those types. The main results are the following.

- For type A I. We will recover the result in Matsumoto (2012) in a a simpler way, which claims that the Weingarten function of type A I is essentially same with the orthogonal Weingarten function.

- For type A III. We will obtain a combinatorial expansion for the A III Weingarten function, as like Theorems 2.7, 4.6.

Our technique can be applied for compact symmetric spaces of remaining types A II, CI, .... However, there are additional technicalities that are intrinsic to any given type. Therefore, in this paper, for the sake of brevity, but yet show the power of the original Weingarten approach, we stick to two types. We expect to handle other types in subsequent research. 
5.1. AI case: COE. Consider the compact symmetric space $\mathrm{U}(d) / \mathrm{O}(d)$. Then the set $\mathbb{S}=\operatorname{COE}(d)$ consists of all $d \times d$ symmetric unitary matrices. The random matrix ensemble $\{\operatorname{COE}(d), d \nu)\}_{d \geq 1}$ is referred to the circular orthogonal ensemble $(\mathrm{COE})$.

Assume $d \geq 2 k$. The Weingarten calculus for the COE is described as follows. For each pair partition $\mathfrak{m} \in \mathcal{P}_{2}(2 k)$, we define the Weingarten function

$$
\mathrm{Wg}^{\mathrm{COE}}(\mathfrak{m}, d)=\int_{\operatorname{COE}(d)} \prod_{j=1}^{k} s_{2 j-1,2 j} \cdot \prod_{\{a, b\} \in \mathfrak{m}} s_{a, b} d \nu .
$$

Note that $s_{a, b}=s_{b, a}$ since a matrix in COE is symmetric. Moreover, for each permutation $\sigma \in \mathcal{S}_{2 k}$, we put

$$
\mathrm{Wg}^{\mathrm{COE}}(\sigma, d)=\mathrm{Wg}^{\mathrm{COE}}\left(\sigma \cdot \mathfrak{e}_{k}, d\right),
$$

where $\sigma \cdot \mathfrak{e}_{k}$ is the pair partition $\{\sigma(1), \sigma(2)\} \cdots\{\sigma(2 k-1), \sigma(2 k)\}$.

Lemma 5.1 (Matsumoto, 2012). For two sequences

$$
\mathbf{i}=\left(i_{1}, \ldots, i_{2 k}\right), \quad \mathbf{j}=\left(j_{1}, \ldots, j_{2 k}\right)
$$

of positive integers in $\{1,2, \ldots, d\}$, we have

$$
\int_{\mathrm{COE}(d)} s_{i_{1}, i_{2}} \cdots s_{i_{2 k-1}, i_{2 k}} \overline{s_{j_{1}, j_{2}} \cdots s_{j_{2 k-1}, j_{2 k}}} d \nu=\sum_{\sigma \in \mathcal{S}_{2 k}} \delta_{\sigma}(\mathbf{i}, \mathbf{j}) \mathrm{Wg}^{\mathrm{COE}}(\sigma, d) .
$$

Here the $\delta$-symbol is defined as in Lemma 2.1.

Lemma 5.2. For any $\sigma, \zeta \in \mathcal{S}_{2 k}$, we have

$$
\mathrm{Wg}^{\mathrm{COE}}\left(\zeta^{-1} \sigma, d\right)=\int_{\mathrm{COE}(d)} s_{\zeta(1), \zeta(2)} \cdots s_{\zeta(2 k-1) \zeta(2 k)} \overline{s_{\sigma(1), \sigma(2)} \cdots s_{\sigma(2 k-1), \sigma(2 k)}} d \nu
$$

Proof: Recall the fact that the probability measure $d \nu$ is invariant under the action of $\mathrm{U}(d)$. Since any permutation matrix is unitary, integrals are invariant under the replacement $\left(s_{i j}\right)_{1 \leq i, j \leq d} \mapsto\left(s_{\zeta(i), \zeta(j)}\right)_{1 \leq i, j \leq d}$. Thus we have the identity

$$
\mathrm{Wg}^{\mathrm{COE}}(\sigma, d)=\int_{\mathrm{COE}(d)} s_{\zeta(1), \zeta(2)} \cdots s_{\zeta(2 k-1) \zeta(2 k)} \overline{s_{\zeta \sigma(1), \zeta \sigma(2)} \cdots s_{\zeta \sigma(2 k-1), \zeta \sigma(2 k)}} d \nu .
$$

Replacing $\sigma$ by $\zeta^{-1} \sigma$, we obtain the desired formula.

We have the following orthogonality relation for $\mathrm{Wg}^{\mathrm{COE}}$.

Lemma 5.3. The Weingarten function $\mathrm{Wg}^{\mathrm{COE}}$ satisfies the following formula: For each $\mathfrak{m} \in \mathcal{P}_{2}(2 k)$,

$$
\begin{aligned}
& (d+1) \mathrm{Wg}^{\mathrm{COE}}(\mathfrak{m}, d) \\
= & -\sum_{i=1}^{2 k-2} \mathrm{Wg}^{\mathrm{COE}}((i, 2 k-1) \cdot \mathfrak{m}, d)+\delta_{\{2 k-1,2 k\} \in \mathfrak{m}} \mathrm{Wg}^{\mathrm{COE}}\left(\mathfrak{m}^{\downarrow}, d\right) .
\end{aligned}
$$

Proof: Consider a pair partition $\mathfrak{m}=\{\mathfrak{m}(1), \mathfrak{m}(2)\} \cdots\{\mathfrak{m}(2 k-1), \mathfrak{m}(2 k)\}$ and suppose $\mathfrak{m}(2 k-1)=2 k-1$. Fix such an expression of $\mathfrak{m}$ and let $\sigma_{\mathfrak{m}}$ be the permutation $j \mapsto \mathfrak{m}(j)$. Consider the sum of integrals

$$
\sum_{i=1}^{d} J_{i}(\mathfrak{m})
$$


with

$$
\begin{aligned}
J_{i}(\mathfrak{m})=\sum_{i=1}^{d} \int_{\operatorname{COE}(d)} & s_{1,2} \cdots s_{2 k-3,2 k-2} s_{i, 2 k} \\
& \times \overline{s_{\mathfrak{m}(1), \mathfrak{m}(2)} \cdots s_{\mathfrak{m}(2 k-3), \mathfrak{m}(2 k-2)} s_{i, \mathfrak{m}(2 k)}} d \nu .
\end{aligned}
$$

Since a matrix in $\operatorname{COE}(d)$ is unitary, we have $\sum_{i=1}^{d} s_{i, 2 k} \overline{s_{i, \mathfrak{m}(2 k)}}=\delta_{\mathfrak{m}(2 k), 2 k}$, and hence (5.1) equals

$$
\begin{aligned}
& \delta_{\mathfrak{m}(2 k), 2 k} \int_{\mathrm{COE}(d)} s_{1,2} \cdots s_{2 k-3,2 k-2} \overline{s_{\mathfrak{m}(1), \mathfrak{m}(2)} \cdots s_{\mathfrak{m}(2 k-3), \mathfrak{m}(2 k-2)}} d s \\
= & \delta_{\{2 k-1,2 k\} \in \mathfrak{m}} \mathrm{Wg}^{\mathrm{COE}}\left(\mathfrak{m}^{\downarrow}, d\right) .
\end{aligned}
$$

On the other hand, using Lemma 5.1 we have

$$
J_{i}(\mathfrak{m})=\left\{\begin{array}{lc}
\mathrm{Wg}^{\mathrm{COE}}\left(\sigma_{\mathfrak{m}}, d\right) & \text { if } i \notin\{1,2, \ldots, 2 k-2,2 k\} \\
\mathrm{Wg}^{\mathrm{COE}}\left(\sigma_{\mathfrak{m}}, d\right)+\mathrm{Wg}^{\mathrm{COE}}(\sigma[\mathfrak{m}, i], d) & \text { if } i \in\{1,2, \ldots, 2 k-2,2 k\},
\end{array}\right.
$$

where $\sigma[\mathfrak{m}, i]$ is the permutation defined by

$$
\begin{aligned}
\sigma[\mathfrak{m}, i] & =\left(\begin{array}{cccccccc}
1 & \ldots & r-1 & r & r+1 & \cdots & 2 k-1 & 2 k \\
\mathfrak{m}(1) & \ldots & \mathfrak{m}(r-1) & 2 k-1 & \mathfrak{m}(r+1) & \ldots & i & \mathfrak{m}(2 k)
\end{array}\right) \\
& =\left(\begin{array}{llll}
i, 2 k-1) \sigma_{\mathfrak{m}} &
\end{array}\right.
\end{aligned}
$$

with $r \in\{1,2, \ldots, 2 k-2,2 k\}$ uniquely determined by $\sigma_{\mathfrak{m}}(r)=i$. If $i=2 k$, we can observe

$$
\mathrm{Wg}^{\mathrm{COE}}\left((2 k-1,2 k) \sigma_{\mathfrak{m}}, d\right)=\mathrm{Wg}^{\mathrm{COE}}\left(\sigma_{\mathfrak{m}}, d\right)
$$

by Lemma 5.2. Summing up them over $i$, we have obtained

$$
\begin{aligned}
(5.1) & =(d+1) \mathrm{Wg}^{\mathrm{COE}}\left(\sigma_{\mathfrak{m}}, d\right)+\sum_{i=1}^{2 k-2} \mathrm{Wg}^{\mathrm{COE}}\left((i, 2 k-1) \sigma_{\mathfrak{m}}, d\right) \\
& =(d+1) \mathrm{Wg}^{\mathrm{COE}}(\mathfrak{m}, d)+\sum_{i=1}^{2 k-2} \mathrm{Wg}^{\mathrm{COE}}((i, 2 k-1) \cdot \mathfrak{m}, d) .
\end{aligned}
$$

Comparing this lemma with Lemma 4.3, we find the fact that the orthogonality relation for $\mathrm{Wg}^{\mathrm{COE}}(\mathfrak{m}, d)$ coincides with that for $\mathrm{Wg}^{\mathrm{O}}(\mathfrak{m}, d+1)$ in association with the shift for $d$. This induces the following theorem immediately.

Theorem 5.4. Suppose $d \geq 2 k$. For any $\mathfrak{m} \in \mathcal{P}_{2}(2 k)$, we have

$$
\mathrm{Wg}^{\mathrm{COE}}(\mathfrak{m}, d)=\mathrm{Wg}^{\mathrm{O}}(\mathfrak{m}, d+1) .
$$

This theorem was first discovered in Matsumoto (2012) by applying harmonic analysis of symmetric groups. In our present proof, we could avoid those involving algebraic discussions. 
5.2. AIII case. Let $a, b$ be positive integers. Put $d=a+b$ and set

$$
d^{-}=a-b .
$$

Let us consider the compact symmetric space $\mathrm{U}(d) /(\mathrm{U}(a) \times \mathrm{U}(b))$ of type A III. The corresponding involution $\theta$ and matrix space $\mathbb{S}$ are $\theta(g)=I_{a b}^{\prime} g I_{a b}^{\prime}$ and $\mathbb{S}=$ $\left\{g I_{a b}^{\prime} g^{*} I_{a b}^{\prime} \mid g \in \mathrm{U}(d)\right\}$, respectively. Here we set

$$
I_{a b}^{\prime}=\operatorname{diag}(\underbrace{1,1, \ldots, 1}_{a}, \underbrace{-1,-1, \ldots,-1}_{b}) .
$$

For convenience, we deal with

$$
\tilde{\mathbb{S}}=\tilde{\mathbb{S}}\left(d, d^{-}\right)=\left\{s=g I_{a b}^{\prime} g^{*} \mid g \in \mathrm{U}(d)\right\}
$$

instead of $\mathbb{S}$. Any matrix in $\tilde{\mathbb{S}}$ is unitary and Hermitian. The induced probability measure $d \nu$ on $\tilde{\mathbb{S}}$ is invariant under the action

$$
G \times \tilde{\mathbb{S}} \ni\left(g_{0}, s\right) \mapsto g_{0} s g_{0}^{*} \in \tilde{\mathbb{S}} .
$$

Suppose that $d \geq k$. The A III Weingarten function is defined by

$$
\mathrm{Wg}^{\mathrm{AIII}}\left(\sigma, d, d^{-}\right)=\int_{\tilde{\mathbb{S}}\left(d, d^{-}\right)} s_{1 \sigma(1)} s_{2 \sigma(2)} \cdots s_{k \sigma(k)} d \nu
$$

for $\sigma \in \mathcal{S}_{k}$. This is a conjugacy-invariant function on $\mathcal{S}_{k}$.

Lemma 5.5 (Matsumoto, 2013). For two sequences

$$
\mathbf{i}=\left(i_{1}, \ldots, i_{k}\right), \quad \mathbf{j}=\left(j_{1}, \ldots, j_{k}\right)
$$

of positive integers in $\{1,2, \ldots, d\}$, we have

$$
\int_{\tilde{\mathbb{S}}\left(d, d^{-}\right)} s_{i_{1} j_{1}} s_{i_{2} j_{2}} \cdots s_{i_{k} j_{k}} d \nu=\sum_{\sigma \in \mathcal{S}_{k}} \delta_{\sigma}(\mathbf{i}, \mathbf{j}) \mathrm{Wg}^{\mathrm{AIII}}\left(\sigma, d, d^{-}\right) .
$$

We introduce an operation $\sigma \mapsto \sigma^{b}$ as follows. Let $k \geq 2$ and suppose that $\sigma \in \mathcal{S}_{k}$ satisfies $\sigma(k)=: r \neq k$ and $\sigma(r)=k$. In other words, the letter $k$ belongs to a 2 -cycle in $\sigma$. If we remove the 2 -cycle $(r, k)$ from $\sigma$, the output is a bijection on $T(r, k)=\{1,2, \ldots, r-1, r+1, \ldots, k-1\}$. We then define the permutation $\sigma^{b}$ in $\mathcal{S}_{k-2}$ by $\sigma^{b}=\left.\iota_{r} \circ \sigma\right|_{T(r, k)} \circ \iota_{r}^{-1}$ with the order-preserved bijection

$$
\iota_{r}: T(r, k) \rightarrow\{1,2, \ldots, k-2\} .
$$

For example, if $\sigma \in \mathcal{S}_{5}$ is

$$
\sigma=\left(\begin{array}{lllll}
1 & 2 & 3 & 4 & 5 \\
4 & 5 & 1 & 3 & 2
\end{array}\right)
$$

in the two-row notation, we have $r=2$ and

$$
\sigma^{b}=\left(\begin{array}{lll}
1 & 2 & 3 \\
3 & 1 & 2
\end{array}\right) \in \mathcal{S}_{3} .
$$

Lemma 5.6. Let $\sigma \in \mathcal{S}_{k}$.

$$
\begin{aligned}
d \mathrm{Wg}^{\mathrm{AIII}}\left(\sigma, d, d^{-}\right)= & -\sum_{i=1}^{k-1} \mathrm{Wg}^{\mathrm{A} \mathrm{III}}\left((i, k) \sigma, d, d^{-}\right) \\
& +\delta_{\sigma(k)=k} d^{-} \mathrm{Wg}^{\mathrm{AIII}}\left(\sigma^{\downarrow}, d, d^{-}\right) \\
& +\delta_{(\sigma(k), k) \in C(\sigma)} \mathrm{Wg}^{\mathrm{AII}}\left(\sigma^{\downarrow}, d, d^{-}\right) .
\end{aligned}
$$


Here, if $\sigma(k) \neq k$ and $\sigma^{2}(k)=k$, i.e, if $k$ belongs to a 2-cycle of $\sigma$, then we set $\delta_{(\sigma(k), k) \in C(\sigma)}=1$.

Proof: First we assume $\sigma(k)=k$ and consider

$$
\sum_{i=1}^{d} \int_{\tilde{\mathbb{S}}} s_{1 \sigma(1)} s_{2 \sigma(2)} \cdots s_{k-1, \sigma(k-1)} s_{i i} d \nu .
$$

Since $\sum_{i=1}^{d} s_{i i}=\operatorname{Tr}(s)=\operatorname{Tr}\left(I_{a b}^{\prime}\right)=a-b=d^{-}$, the sum (5.4) equals $d^{-} \operatorname{Wg}\left(\sigma^{\downarrow}, d, d^{-}\right)$. On the other hand, as in the unitary case, we see that

$$
\begin{aligned}
& \int_{\tilde{\mathbb{S}}} s_{1 \sigma(1)} \cdots s_{k-1, \sigma(k-1)} s_{i i} d \nu \\
= & \begin{cases}\operatorname{Wg}^{\mathrm{A} I I I}\left(\sigma, d, d^{-}\right) & \text {if } i \geq k, \\
\operatorname{Wg}^{\mathrm{AIII}}\left(\sigma, d, d^{-}\right)+\mathrm{Wg}^{\mathrm{AIII}}\left((i, k) \sigma, d, d^{-}\right) & \text {if } i<k\end{cases}
\end{aligned}
$$

by Lemma 5.5, and hence

$$
(5.4)=d \mathrm{Wg}^{\mathrm{A} I \mathrm{II}}\left(\sigma, d, d^{-}\right)+\sum_{i=1}^{k-1} \mathrm{Wg}^{\mathrm{A} \mathrm{III}}\left((i, k) \sigma, d, d^{-}\right) .
$$

Thus we have obtained the desired equality for the case where $\sigma(k)=k$.

Next we assume $\sigma(k) \neq k$ and let $r=\sigma^{-1}(k) \in\{1,2, \ldots, k-1\}$. Consider

$$
\sum_{i=1}^{d} \int_{\tilde{\mathbb{S}}} s_{1 \sigma(1)} \cdots s_{r-1, \sigma(r-1)} s_{r, i} s_{r+1, \sigma(r+1)} \cdots s_{k-1, \sigma(k-1)} s_{i, \sigma(k)} d \nu .
$$

(Note that each term is obtained from $s_{1 \sigma(1)} \cdots s_{r k} \cdots s_{k \sigma(k)}$ by replacing two $k$ 's with $i$.) Since

$$
\sum_{i=1}^{d} s_{r i} s_{i t}=\sum_{i=1}^{d} s_{r i} \overline{s_{t i}}=\delta_{r t}
$$

the sum (5.5) equals

$$
\begin{aligned}
& \delta_{r \sigma(k)} \int_{\tilde{\mathbb{S}}} s_{1 \sigma(1)} \cdots s_{r-1, \sigma(r-1)} s_{r+1, \sigma(r+1)} \cdots s_{k-1, \sigma(k-1)} d \nu \\
= & \delta_{(r, k) \in C(\sigma)} \mathrm{Wg}^{\mathrm{AIII}}\left(\sigma^{b}, d, d^{-}\right)
\end{aligned}
$$

by the definition of $\sigma^{b}$. On the other hand, it is easy to see that (5.5) equals

$$
d \mathrm{Wg}^{\mathrm{A} \mathrm{IIII}}\left(\sigma, d, d^{-}\right)+\sum_{i=1}^{k-1} \mathrm{Wg}^{\mathrm{A} \mathrm{III}}\left((i, k) \sigma, d, d^{-}\right)
$$

by Lemma 5.5 again. This completes the proof of the lemma.

Let us consider the Weingarten graph $\mathcal{G}^{\mathrm{A}} \mathrm{III}=(V, E)$ of type A III.

- The vertex set $V$ is $\bigsqcup_{k=0}^{\infty} \mathcal{S}_{k}$. Each vertex $\sigma$ in $\mathcal{S}_{k}$ is said to be of level $k$.

- For each $k \geq 2$, two vertices $\sigma, \tau$ of level $k$ are connected by a solid arrow if

$$
\tau=(i, k) \sigma \quad \text { with some } i \text { smaller than } k .
$$

We write $\sigma \rightarrow \tau$.

- For each $k \geq 1$, a vertex $\sigma$ of level $k$ and a vertex $\sigma^{\prime}$ of level $k-1$ are connected by a dashed arrow if $\sigma(k)=k$ and $\sigma^{\prime}=\sigma^{\downarrow}$. We write $\sigma \rightarrow \sigma^{\prime}$. 
- For each $k \geq 2$, a vertex $\sigma$ of level $k$ and a vertex $\sigma^{\prime \prime}$ of level $k-2$ are connected by a squiggled arrow if $\sigma(k)=: r \neq k$ and $\sigma(r)=k$, and moreover $\sigma^{\prime \prime}=\sigma^{b}$. We write $\sigma \rightsquigarrow \sigma^{\prime \prime}$.

For example, as already observed, we have an squiggled arrow

$$
\mathcal{S}_{5} \ni\left(\begin{array}{lllll}
1 & 2 & 3 & 4 & 5 \\
4 & 5 & 1 & 3 & 2
\end{array}\right) \rightsquigarrow\left(\begin{array}{ccc}
1 & 2 & 3 \\
3 & 1 & 2
\end{array}\right) \in \mathcal{S}_{3} .
$$

A vertex $\sigma \in \mathcal{S}_{k}$ has exactly $k-1$ solid edges and at most 1 dashed edge and at most 1 squiggled edge. No vertex has both dashed edges and squiggled edges.

Let $\sigma \in \mathcal{S}_{k}$ and consider a path $p$ from $\sigma$ to $\emptyset$ in $\mathcal{G}^{\text {A III }}$ as usual. But in this case, there are squiggled edges. Denote by

$$
\ell_{0}(p), \quad \ell_{1}(p), \quad \ell_{2}(p)
$$

the numbers of solid/dashed/squiggled edges such that $p$ gets through, respectively. It is clear that

$$
\ell_{1}(p)+2 \ell_{2}(p)=k
$$

Put $\ell(p)=\ell_{0}(p)+\ell_{1}(p)+\ell_{2}(p)$.

Due to Lemma 5.6 it is not difficult to see the following theorem.

Theorem 5.7. Let $\sigma \in \mathfrak{S}_{k}$. Then

$$
\mathrm{Wg}^{\mathrm{A} \mathrm{III}}\left(\sigma, d, d^{-}\right)=\sum_{p: \sigma \rightarrow \emptyset}(-1)^{\ell_{0}(p)}\left(d^{-}\right)^{\ell_{1}(p)} d^{-\ell(p)} .
$$

Example 5.8. Consider $\sigma=[2,1] \in \mathcal{S}_{2}$. There are two kinds of paths from $\sigma$ to $\emptyset$ :

$$
\begin{aligned}
& {[2,1] \rightarrow[1,2] \rightarrow[2,1] \rightarrow[1,2] \rightarrow \cdots \rightarrow[2,1] \rightsquigarrow \emptyset} \\
& {[2,1] \rightarrow[1,2] \rightarrow[2,1] \rightarrow[1,2] \rightarrow \cdots \rightarrow[1,2] \rightarrow[1]-\rightarrow \emptyset .}
\end{aligned}
$$

The first one contributes to the term $d^{-(2 l+1)}$ and the second one contributed to $-\left(d^{-1}\right)^{2} d^{-(2 l+3)}$. Thus we have the expansion

$$
\mathrm{Wg}^{\mathrm{AIII}}\left([2,1], d, d^{-}\right)=\sum_{l \geq 0} d^{-(2 l+1)}+\sum_{l \geq 0}(-1)\left(d^{-}\right)^{2} d^{-(2 l+3)}
$$

which equals $\frac{d^{2}-\left(d^{-}\right)^{2}}{d\left(d^{2}-1\right)}$.

In Matsumoto (2013), we obtained the Fourier expansion

$$
\mathrm{Wg}^{\mathrm{A} \text { III }}\left(\sigma, d, d^{-}\right)=\frac{1}{k !} \sum_{\lambda \vdash k} \frac{s_{\lambda}\left(1^{a},(-1)^{b}\right)}{s_{\lambda}\left(1^{d}\right)} \chi^{\lambda}(\sigma),
$$

where $s_{\lambda}=s_{\lambda}\left(x_{1}, \ldots, x_{d}\right)$ is the Schur function and $\chi^{\lambda}$ is the irreducible character of symmetric groups. We know the formula $s_{\lambda}\left(1^{d}\right)=\frac{f^{\lambda}}{k !} \prod_{(i, j) \in \lambda}(d+j-i)$, but there is no such closed formula for $s_{\lambda}(\underbrace{1,1, \ldots, 1}_{a}, \underbrace{-1,-1, \ldots,-1}_{b})$. Theorem 5.7 gives a new combinatorial expression for the A III Weingarten function.

\section{Acknowledgements}

Both authors would like to thank an anonymous referee for very constructive comments on the first version of the manuscript. BC acknowledges useful discussions with Mike Brannan. 


\section{References}

F. Benaych-Georges. Exponential bounds for the support convergence in the single ring theorem. J. Funct. Anal. 268 (11), 3492-3507 (2015). MR3336731.

G. Berkolaiko and J. Kuipers. Combinatorial theory of the semiclassical evaluation of transport moments. I. Equivalence with the random matrix approach. J. Math. Phys. 54 (11), 112103, 26 pp. (2013). MR3137025.

M. Brannan and B. Collins. Dual bases in Temperley-Lieb algebras, quantum groups, and a question of Jones. ArXiv Mathematics e-prints (2016). arXiv: 1608.03885.

E. Cartan. Sur certaines formes Riemanniennes remarquables des géométries à groupe fondamental simple. Ann. Sci. École Norm. Sup. (3) 44, 345-467 (1927). MR1509283.

B. Collins. Moments and cumulants of polynomial random variables on unitary groups, the Itzykson-Zuber integral, and free probability. Int. Math. Res. Not. (17), 953-982 (2003). MR1959915.

B. Collins, C. E. González-Guillén and D. Pérez-García. Matrix product states, random matrix theory and the principle of maximum entropy. Comm. Math. Phys. 320 (3), 663-677 (2013). MR3057186.

B. Collins and P. Śniady. Integration with respect to the Haar measure on unitary, orthogonal and symplectic group. Comm. Math. Phys. 264 (3), 773-795 (2006). MR2217291.

B. Collins and M. Stolz. Borel theorems for random matrices from the classical compact symmetric spaces. Ann. Probab. 36 (3), 876-895 (2008). MR2408577.

V. Féray. On complete functions in Jucys-Murphy elements. Ann. Comb. 16 (4), 677-707 (2012). MR3000438.

I. P. Goulden, M. Guay-Paquet and J. Novak. Monotone Hurwitz numbers in genus zero. Canad. J. Math. 65 (5), 1020-1042 (2013). MR3095005.

S. Matsumoto. Jucys-Murphy elements, orthogonal matrix integrals, and Jack measures. Ramanujan J. 26 (1), 69-107 (2011). MR2837720.

S. Matsumoto. General moments of matrix elements from circular orthogonal ensembles. Random Matrices Theory Appl. 1 (3), 1250005, 18 pp. (2012). MR2967964.

S. Matsumoto. Weingarten calculus for matrix ensembles associated with compact symmetric spaces. Random Matrices Theory Appl. 2 (2), 1350001, 26 pp. (2013). MR3077830.

S. Matsumoto and J. Novak. Jucys-Murphy elements and unitary matrix integrals. Int. Math. Res. Not. IMRN (2), 362-397 (2013). MR3010693.

A. Montanaro. Weak multiplicativity for random quantum channels. Comm. Math. Phys. 319 (2), 535-555 (2013). MR3037588.

H. Robbins. A remark on Stirling's formula. Amer. Math. Monthly 62, 26-29 (1955). MR0069328.

S. Samuel. U $(N)$ integrals, $1 / N$, and the De Wit-'t Hooft anomalies. J. Math. Phys. 21 (12), 2695-2703 (1980). MR597583.

A. Soshnikov. Universality at the edge of the spectrum in Wigner random matrices. Comm. Math. Phys. 207 (3), 697-733 (1999). MR1727234.

D. Weingarten. Asymptotic behavior of group integrals in the limit of infinite rank. J. Mathematical Phys. 19 (5), 999-1001 (1978). MR0471696. 
P. Zinn-Justin. Jucys-Murphy elements and Weingarten matrices. Lett. Math. Phys. 91 (2), 119-127 (2010). MR2586868. 